\title{
Valeurs bromatologiques de 150 aliments de l'Ouest Africain
}

\author{
par B. MONGODIN ef R. RIVIÈRE
}

\begin{abstract}
RÉSUMÉ
Un certain nombre de produits originaires des pays de l'Afrique de l'Ouest francophones, susceptibles d'être utilisés en alimentation animale, ont été analysés et étudiés en considérant les points de vue technique, économique et social de leur utilisation.

Ces produits appartiennent aux calégories suivantes :

grains et dérivés,

autres végétaux essentiellement glucidiques,

graınes oléagineuses ef leurs sous-produits,

produits végétaux divers,

produits d'orıgıne animale.

Cette étude doit permettre un calcul plus précis des rations.
\end{abstract}

\section{I. - INTRODUCTION}

Un certain nombre de produits susceptibles d'être donnés comme aliments aux animaux domestıques ont été prélevés dans les différents pays de l'Afrique de l'Ouest francophone.

Ils ont été examinés en considérant les points de vue technıque, économique et social de leur utilisation et des renseignements en ont été tirés qui peuvent intéresser le praticien chargé, sur place, de nourrir des anımaux.

L'objet de cette étude est, d'une part, de permettre un calcul plus précis des rations, et, d'autre part, de faciliter l'évaluation des bılans des productions locales intéressant l'alimentation du bétall.

Elle a eu, en outre, pour résultat de mettre en évidence la très grande varıabilıté des valeurs bromatologiques des matières examinées. Les prodults européens, de catégorie similaire, ont une composition beaucoup plus régulière; cette régularité, indispensable pour le fabricant d'alıments concentrés, a même tendance, actueliement, à prendre un caractère de «standardısation réglementée».

L'examen des différences, entre les productions africaines et celles des pays industrialisés n'entre pas dans le cadre de cette étude. Nous attirons simplement l'attention des utilisateurs et leur conseillons d'être prudents, sınon réservés, autrement dit, de ne pas se laisser tenter par le «dogmatısme» qui prend naissance en cette matıère dans les pays européens.

Au contraire, la nécessité à la fols sociale, politıque et économıque d'utilıser au maximum les sous-produits d'origine locale, impose aux techniciens œuvrant en Afrique de se dégager des conceptions de l'Industric Européenne des Allments de Bétail ; ils doivent s'engager hardiment dans la formulation de règles et de technıques propres au milieu dans lequel et paur lequel ils travaillent. 


\section{II. - GÉNÉRALITÉS}

Les analyses, dans la majorité des cas, ont été effectuées au Laboratorre d'Alımentation et Nutrition de I'I. E. M.V.T., sur des produits reçus des pays de l'Afrique Occidentale.

Les méthodes d'analyse utilısées pour la plupart des dosages, mettent en œuvre les techniques officielles fixées par arrêté du 7 novembre 1960, approuvées par le Service de la Répression des Fraudes.

L'Institut National Agronomıque,

L'Institut Professionnel de contrôle ef de recherches scientifiques des industries de l'alimentation animale ef l'Association Française de Zootechnie.

Pour le dosage de la cellulose et du calcium, des méthodes différentes ont, toutefois, dû être adoptées, par suite de la nécessıté de leur exécutıon en série.

La méthode de SHARRER a été choisie pour la cellulose (hydrolyse par un mélange d'acides acétique, nitrique et trichloracétique) et la méthode complexométrique pour le calcium (sel disodique de l'E. D. T. A. avec la calcéine comme indicateur).

La matière grasse est dosée par extraction à l'éther sulfurique pendant $6 \mathrm{~h}$, les matières protéiques totales par la méthode de KJELDAHL et le phosphore par la méthode colorimétrique de MISSION (complexe jaune de phosphovanadomolybdate d'ammonium).

\section{III. - CALCUL DES COEFFICIENTS DE DIGESTIBILITÉ ET DES ÉNERGIES}

On sait qu'un même aliment est exploité très différemment suivant les espèces animales. Nous avons fait les calculs pour les plus représentatives de l'Ouest africain : bovins adultes, porcins ef poulets. On verra que les différences de valeur bromatologique d'un même aliment sont quelquefois très importantes suivant qu'il est donné à un bovin, ou à un porc par exemple.

Ces différences doivent être prises en considération dans l'économie de l'Elevage, lorsque celle-ci est examinée à l'échelon national ou régional : suivant que tel ou tel sous-produit domine, on pourra prendre une option sur l'es- pèce qui transforme le mieux la matière qui sert de base à l'alimentation.

Les coefficients de digestibilité ont été tirés des tables de SCHNEIDER pour les bovins et les porcins; de MORRISSON pour les bovins et de TITUS pour les volailles.

Pour les problèmes de rationnement de l'Ouest africaIn, les tables de SCHNEIDER donnent des renseignements intéressants parce que cet auteur a utilisé les résultats d'essaıs de digestibilité réalısés par de nombreux chercheurs sous des climats tropicaux ou subtropicaux avec des aliments d'origine locale et des animaux de races locales. Pour les bovins, nous avons utilisé les tables de MORRISSON (FEEDS and FEEDING) lorsque nous n'avons pas trouvé exactement le même aliment dans celles de SCHNEIDER.

On rencontrera, dans les tableaux qui suivent, de nombreux chiffres suivis d'un point d'interrogation; ce signe indique que nous n'avons pas trouvé, dans les tables citées, l'aliment considéré, ou que nous étions en présence du même aliment, mais avec une composition très différente (par exemple, gros sons de blé pour les volalles, lorsqu'ils ont une forte teneur en cellulose). Dans ce cas, les calculs ont été effectués par assimilation à des matıères d'origine semblable et du même genre botanique. Les chiffres donnés devront donc être utilısés avec prudence, en se donnant une «fourchette de sécurité ».

Enfin, certaines cases de ces tables sont vides de chiffres. Cela signifie que l'aliment n'est pas donné à l'espèce envisagée dans les conditions habituelles d'élevage (exemple : levure pour les bovins adultes), au que nous n'avons pas trouvé d'équivalent qui puisse nous permettre de calculer les valeurs bromatologiques avec une approximation utilisable (exemple: farine de banane pour les porcs et les volailles). "Dans ce dernier cas, il y a un problème technique à résoudre et il peut falre l'objet d'un travall de recherche appliquée dont l'utılıté, du point de vue économıque et social, peut être importante.

Les valeurs énergétiques ont été calculées en T.D.N. (total digestible nutrients), en U.F. (unités fourragères) et en E.M. (énergie métabolisable, exprimée en grandes calories).

Tous ces calculs sont basés sur deux éléments essentiels : la composition en principes alimentaires, et le coefficient de digestibilité propre à chacun de ces principes et à l'espèce animale 
envisagée. La composition est donnée par l'analyse chimique. Nous y avons inclus systématıquement l'insoluble chlorhydrique dont le taux est quelquefols très élevé lorsque les aliments sont préparés localement dans des conditions que nous examinerons dans chaque cas particulier.

Les coefficients de digestibılité ont été tirés, nous l'avons déjà dit, des tables de SCHNEIDER, MORRISSON et TITUS. Dans les calculs, ils sont affectés de facteurs dépendant à la fois de l'aliment et de l'espèce et qui ont été déterminés expérimentalement d'après des essais effectués par de nombreux auteurs et plus particulièrement LEROY, FRAPS, TITUS, BOLTON.

Les T.D.N. sont surtout utilisés aux Ełats-Unıs pour les porcs ef les bovins.

Ils sont calculés pour $100 \mathrm{~g}$ d'aliment et correspondent à la formule suivante :

T.D.N. = M.P.D. + M. C. D. + E. N. A. D. $+(2,25 \times$ M. G. D. $)$.

- M.P.D. : Matières protéiques digestibles

- M.C.D. : Matières cellulosiques digestibles

Contenus dans

- E. N. A. D. : Extractif non azoté digestıble

- M.G.D. : Matières grasses digestibles

$100 \mathrm{~g}$ d'aliment

Les unités fourragères (U. F.) et l'Energie métabolisable ( $E . M_{1}$ )

La valeur fourragère d'un alıment est la quantité d'orge exprimée en $\mathrm{kg}$ qui produirait, pour l'espèce envisagée, le même effet énergétique qu'un $\mathrm{kg}$ de cet aliment.

Cette méthode est surtout utilisée, en France, pour les bovins ef les porcıns; elle est, à notre avis, surtout pour les aliments très grossiers, beaucoup plus valable que les T.D.N., parce qu'on tient compte dans son calcul de l'énergie perdue au cours de la digestion (énergie de consommation).

$$
U . F .=\frac{E . N .}{E \cdot O .}
$$

E. N. : énergıe nette dans $1 \mathrm{~kg}$ d'alıment.

E. O. : énergie dans un $\mathrm{kg}$ d'orge.

Nous avons pris :

E. O. $=2.350$ pour les porcs.

E. O. $=1,880$ pour les bovins.

$$
\text { E. N. = E.M. - E. C. }
$$

E. $M$. = énergie métabolisable dans $1 \mathrm{~kg}$ d'aliment.

E. C. = énergie de consommation.

E. M. $=$ T. D. N. $\times \mathrm{k} \times 10$

$k$ (équivalent calorique) $=3,65$ pour les ruminants.

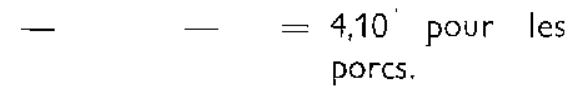

E. C. $=$ M.S. $\times \mathrm{k}^{\prime}$

$\mathrm{k}^{\prime}=1,00$ pour les ruminants.

$=0,85$ pour les porcs.

M. S. = matière sèche dans $1 \mathrm{~kg}$ d'aliment.

L'énergle métabolisable esł utilisée pour les porcins et les volailles.

Les coefficients d'équivalence calorique affectés aux prıncipes digestibles pour cette dernière espèce sont les suivants :

- Matières azotées $\left(k_{1}\right)=3,84$.

- Matières grasses $\left(k_{2}\right)$

$=9,49$ pour les farines animales.

$=9,33$ pour les graıns de céréales et autres graines.

$=9,21$ pour les dérıvés du lait.

- E. N.A. $\left(k_{3}\right)$

$=4,2$ pour les grains.

$=4,0$ pour les graines.

$=3,8$ pour les fevilles.

$=3,7$ pour les dérivés du lait.

- Celiulose $\left(k_{4}\right)=2,1$ :

d'où énergie métabolisable pour volalles, toujours calculée pour $1 \mathrm{~kg}$ d'alıment :

E. M. $=k_{1} M \cdot P \cdot B \cdot c d_{1}$ Les quantités des prın-

$+k_{2} M . G . c_{2}$ cipes alımentaires sont $+k_{3}$ E. N. A. $c_{3}\left\{\begin{array}{l}\text { celles contenues dans } \\ 1 \mathrm{~kg} \text { d'alıment. }\end{array}\right.$

$+k_{4}$ M.C. D. $\mathrm{cd}_{4}$ ) cd est le coefficient de digestıbılité du principe alımentaire correspondant chez les poulets.

\section{IV. - ANALYSES \\ VALEURS BROMATOLOGIQUES COMMENTAIRES}

\section{A. - LES GRAINS \\ ET LEURS DÉRIVÉS}

Petit mil (Tableav I).

Il s'agit là du petit mil, mil pénicıllaire, appelé aussi mıl chandelle, mıl d'Egypte; espèce : 
TARLEAU N $N^{\circ}$ I

Mi1 pemicillaire or petit mil

\begin{tabular}{|c|c|c|c|c|}
\hline & 1 & 2 & 3 & 4 \\
\hline Eumidité & 12,35 & 12,4 & 10,79 & 7,26 \\
\hline Matière sèche & 87,65 & 87,6 & 89,28 & 92,74 \\
\hline M.P.B. & 9,02 & 8,9 & 10,13 & 12,12 \\
\hline Cellulose & 2,00 & 1,4 & 0,60 & 2,10 \\
\hline Kat- grasges & 4,38 & 4,75 & 5,49 & 5,20 \\
\hline Mat. min. & 2,24 & 1,65 & 2,06 & 1,52 \\
\hline Calcium & 0,025 & 0,020 & 0,020 & 0,026 \\
\hline Phosphore & 0,268 & 0,300 & 0,213 & 0,267 \\
\hline Ins. chl. & 0,67 & & 0,80 & 0,12 \\
\hline E.N.A. & 70,01 & 70,90 & 71,00 & 71,74 \\
\hline \multicolumn{5}{|l|}{ M.P.D. Bovin } \\
\hline M.P.D. Porc & 5,50 & 5,43 & 6,18 & 7,39 \\
\hline M.P.D. Volaille & $6,85 ?$ & $6,76 ?$ & $7,70 ?$ & $9,21 ?$ \\
\hline \multicolumn{5}{|l|}{ T.D.N. Bovin } \\
\hline T.D.N. Pore & 55,69 & 56,47 & 58,04 & 59,83 \\
\hline \multicolumn{5}{|l|}{ J. F. Bovin } \\
\hline U. F. Porc & 0,65 & 0,66 & 0,69 & 0,71 \\
\hline E. N. Pore & 2283 & 2315 & 2379 & 2453 \\
\hline E. M. Volaille & $3147 ?$ & $3201 ?$ & $3291 ?$ & $3361 ?$ \\
\hline
\end{tabular}

1. Petit mil (Haute Côte d'Ivoire) - 2. Petit mil (Haute Volta) - 3. Petit mil Maradi (Niger) - 4. Petit mil Tillabery (Niger)

Pennisetum Typhoidum ou Glaucum ou Sprcatum ou Americanum. On le confond souvent en France avec d'autres grains qu'on appelie mils, petits mils, mils de Hongrie, etc... qui sont de genres différents. En Anglais, tous ces grains sont appelés «Millet» (même certains sorghos) ce qui ajoute à la confusion.

La précision dans l'appellation est importante, car ils sont loin d'avoir une valeur alimentaire équivalente et le petıt mił (Pennisetum Glaucum) se distıngue partıculièrement par ses qualités nettement en retrait des sorghos par exemple.

En Anglais, c'est « pearl millet» et les tables de SCHNEIDER dannent, à cette appellation, des coefficients de digestibilité déterminés chez le parc après deux séries d'essals. C'est à partır de ces données qu'ont été calculées les valeurs énergétiques ci-contre. On voit que ce petit mil, avec des valeurs en U. F. comprises entre 0,65 et 0,71 , est nettement en dessous des sorghos $(1,02$ à 1,05$)$ et des mals $(1,00$ à 1,15).

En appliquant au petit mil les coefficients de digestibilité du sorgho, la valeur énergétıque qurait été comprise, pour le porc, entre 1,02 ef 1,05 U. F. Soit une erreur de 45 à 60 p. 100 par rapport à la valeur réelle! On volt là le danger des extrapolations ef assimilations en alımentation animale.

Pour les poulets, nous n'avons trouvé aucune donnée sûre. Les tables de digestibilités de langue anglaise, se rapportant à cette espèce, donnent des résultats d̀ l'appellation «Millet», sans autre 
précision. Il s'agit probablement du mil de Hongrie (foxtail millet) Setaria itolica. Les chiffres donnés $\mathrm{Cl}$-contre ont été calculés à partir de ce «Millet». Ils doivent être utilisés avec beaucoup de circonspection.

Nous pensons que la moindre valeur énergétique du petit mil vient de son fort pourcentage d'enveloppes qui augmente nettement l'indigestible glucidique et diminue aussi les coefficients de digestibilité des autres principes alimentaires.

\section{Sorgho ef Sons (Tableau II).}

Il y a beaucoup moins de confusion dans les appellations se rapportant aux sorghos que dans celles des petits mils.

Les sorghos correspondent à un seul genre botanique: Sorghum, avec plusieurs espèces : Vulgare, Technicum, etc... Les échantillons que nous avons fait analyser correspondent aux noms français de sorgho, gros mil, mil d'Afrique; en anglais, great millet, milo, american broom corn, etc...

Au point de vue bromatologique, les sorghos ont une composition volsine de celle des petits mils; mais la valeur alimentaire pour le porc, en particulier, est nettement supérieure. Tous les chiffres que nous rapportons dans les cinq premières colonnes du tableau II, correspondent à des essais effectués sur des sorghos en Afrique de l'Est. On notera, fait assez rare, que ces grains ont une valeur énergétique légèrement supérieure pour les poulets. Ils peuvent être, pour ceux-ci, assimilés aux maïs, en notant toutefols,

TABILEAU NO II

Sorgho et son

\begin{tabular}{|c|c|c|c|c|c|c|}
\hline & 1 & 2 & 3 & 4 & 5 & 6 \\
\hline Gumindiété & 9.22 & 12.32 & 0,75 & 9,14 & 10,09 & 9,82 \\
\hline Mat1ère sèche & 90,78 & 87,68 & 91,25 & 30.36 & 89,31 & 90,15 \\
\hline M.P.B. & 8,96 & 8,93 & $\$ 3,45$ & 10,32 & 10,48 & 13,28 \\
\hline Cellulose & 2,10 & 2,14 & 3,20 & 2,30 & 2,80 & 4,26 \\
\hline Mat. Fraseas & 3.10 & 3,00 & 2,70 & 3,96 & 3,40 & 3,46 \\
\hline Het, 毗. & 3,40 & 1.56 & 1,85 & 1,72 & 1,60 & 3.55 \\
\hline calotivm & 0,041 & 0,012 & 0,028 & 0.028 & 0.024 & 0,050 \\
\hline Phosphore & 0,266 & 0,28 & 0,362 & 0,344 & 0,203 & 0,460 \\
\hline Ins. chl. & 1,19 & 0,11 & 0,15 & 0,14 & 0,36 & 0,97 \\
\hline E.N.A. & 73,22 & 72,05 & 70,05 & 71.96 & 71,03 & 51,17 \\
\hline M.P.D. Boorn & 5,11 & 5,09 & 7,66 & 5,88 & 3,97 & $10,36 ?$ \\
\hline M.P.D. Porc & 6,36 & 6,34 & 9,55 & 7.33 & 7.44 & $10,09 ?$ \\
\hline M.P.D. Vola1lle & 7,53 & 7,50 & 11,30 & 8,67 & $B, a 0$ & $8,76 ?$ \\
\hline T.D.N. Bovin & 72,30 & 71,94 & 71,94 & 73,69 & 72,17 & $69,86 ?$ \\
\hline T.D.N. Porc & 77,83 & 76,64 & 78,35 & 79,35 & 77,80 & $64,27 ?$ \\
\hline D. F. Bovin & 0,92 & 0,91 & 0,91 & 0,95 & 0,92 & $0,87 ?$ \\
\hline U. I. Porc & 1,03 & 1,02 & 1,04 & 1,05 & 1,03 & $0,79 ?$ \\
\hline E. M. Porc & 3191 & 3142 & 3212 & 3253 & 3190 & $2635 ?$ \\
\hline פ. M. Volaille & 3389 & 3336 & 3384 & 3451 & 3377 & $1883 ?$ \\
\hline
\end{tabular}

1. Sorgho Cotonou (Dahomey) - 2. Sorgho Lome (Togo) - 3. Sorgho Sotuba (Mal1) - 4. Sorgho Sotube (Mali) 5. Sorgho Meradi (Niger) - 6. Son de gros mil-Tillobery (Niger) 
qu'ils sont toujours très pauvres en vitamine et provitamine $A$.

En colonne 6, on trouvera la composition d'un son de gros mil provenant d'un petit moulin artisanal. Nous n'avons pu malheureusement recueillir le sorgho dant était issu ce son.

En conclusion pratique, on utilisera de préférence les sorghos aux petits mils pour l'alimentation des animaux domestıques ef plus particulièrement des porcs et des poulets.

Les zones de culture de ces deux céréales se confondent en partie et on a souvent le choix entre les deux; même à un prix plus élevé, on achètera du sorgho.

Le mä̈s (Tableaux Ilt et IV)

THBLFHU YO० III

Tikĩs-trrains de pays secs

\begin{tabular}{|c|c|c|c|}
\hline & 1 & 2 & 3 \\
\hline Humidi té & 6,40 & 9,90 & 7,19 \\
\hline Hatière sèche & 93,60 & 90,10 & 92,81 \\
\hline$M . P . B$. & 11,62 & 11,32 & 9,34 \\
\hline Cellulose & 2,30 & 2,27 & 2,75 \\
\hline Fat. grasses & 3,65 & 3,93 & 3,73 \\
\hline Mat. min. & 1,72 & 1,83 & 1,41 \\
\hline Calciurn & 0,015 & 0,026 & 0,025 \\
\hline Phosphore & 0,370 & 0.344 & 0,305 \\
\hline Ins. chl. & 0,09 & 0.17 & 0,10 \\
\hline E.N.A. & 74.31 & 70,75 & 75,58 \\
\hline M.P.D. Bovin & 3,71 & 8,49 & 7,00 \\
\hline M.P.D. Pore & 9,29 & 9,06 & 7,47 \\
\hline M.P.D. Volaille & 8,83 & 8,60 & 7,10 \\
\hline T.D.N. Bovin & 83,89 & 80,96 & 83,57 \\
\hline T.D.N. Pore & 85,24 & 82,12 & 84,90 \\
\hline U. F. Bovin & 1,08 & 1.09 & 1,07 \\
\hline D. Fn Poro & 1,15 & 1,11 & 1,15 \\
\hline E. M. Pore & 3495 & 3366 & 3481 \\
\hline E. M. Volaille & 3447 & 3325 & 3436 \\
\hline
\end{tabular}

1. Maradi (Niger) - 2. Tillabery (Niger) - 3. Sotuba (Mali)
Cette céréde se cultıve dans les zones à clımats guinéen, soudano-guinéen et soudanien. Mais les grains qui en sont issus se distinguent nettement survant qu'ils ont été récoltés en pays humide ou sec.

Les différences portent essentiellement sur la teneur en matières protéiques et sur la consistance.

Les chiffres que nous avons rapportés montrent nettement que la teneur en M.P. B. augmente lorsqu'on s'éloigne des régıons côtières; cette relation est confirmée par les teneurs des mals provenant des zones intermédiaires (Haute Côte-d'lvoire : - colonne 1 du tableau $\mathrm{V}$ ef Nord Dahomey: - colonne 4 du même tableau); ces teneurs sont, elles aussi, intermédıaires entre celles des pays très humides et celles des pays secs. D'autres résultats que nous n'avons pas relevés dans ces tableaux confirment ces observations.

La consistance des grains est aussı variable : ceux des pays proches de la mer sont tendres ef ceux des pays secs sont plus petits et surtout plus durs: on qualifie ces derniers de cornés ou vitreux. Cet aspect particulier est dû à la présence de cellules à aleurone situées à la périphérie des grains. "L'aleurone est un complexe protéique, noyé dons un réseau de matières grasses, fortement minéralisé » (ADRIAN). II y a donc une corrélation entre la dureté et la richesse en matières protéıques, dues toutes les deux, à la présence de l'aleurone.

Les différences de taux de matières protéiques sont quelquefois très grandes : jusqu'à 70 p. 100 en plus par rapport au taux le moins élevé. C'est un facteur important à considérer dans les calculs de rationnement pour les volailles.

La dureté des grains présente un avantage pour le stockage : ils sont très résistants aux insectes. Des pertes consıdérables sont enregıstrées dans le stackage des grains tendres (30 à 50 p. 100 : observations effectuées au Bas Dahomey), alors que les grains que nous avons examinés au Mali et au Niger après 3 à 4 mols de stockoge, étaient indemnes de toute attaque d'insectes prédateurs.

Mais les grains cornés doivent être concassés avant d'être donnés au bétail car les enveloppes résistent aux attaques des sucs digestıfs,

D'où vient l'abondance des cellules à aleurone que l'on trouve dans certains grains ? On a pensé 
TARIPAU NO IV

MaIs-zrains de pays cottiers

\begin{tabular}{|c|c|c|c|c|c|c|}
\hline & 1 & 2 & 3 & 4 & 5 & 6 \\
\hline Huniditế & 14,85 & 12,00 & 11,25 & 12,09 & 15,02 & 12,36 \\
\hline Matière sèche & 85,15 & 88,00 & 88,75 & 87,91 & 84,98 & 87,64 \\
\hline M.P.B. & 10,43 & 6,93 & 9,05 & 10,17 & 7,98 & 8,36 \\
\hline Ceilulose & 1,87 & 1,58 & 1,55 & 1,83 & 2,73 & 1,42 \\
\hline Mat. grasses & 2,94 & 2,97 & 3,75 & 3,70 & 0,68 & 3,77 \\
\hline Mat. min. & 1,27 & 1,41 & 1,40 & 1,41 & 1.70 & 1,44 \\
\hline Calciun & 0,051 & 0,070 & 0,015 & 0,014 & 0,030 & 0,012 \\
\hline Phosphore & 0,306 & 0,378 & 0,300 & $0,6,10$ & 0,160 & 0,290 \\
\hline Ing. ch1. & & & & 0,03 & 0.25 & 0,12 \\
\hline E.N.A. & 68,62 & 75,10 & 73,00 & 70,80 & 72,03 & 72,05 \\
\hline M.P.D. Bovin & 7,82 & 5,20 & 6,79 & 7,63 & 5,98 & 6,27 \\
\hline M.P.D. Porc & 8,34 & 5,54 & 7,24 & 8,14 & 6,34 & 6,69 \\
\hline M.P.D. Volaille & 7,93 & 5,26 & 6,88 & 7,73 & 6,06 & 6,35 \\
\hline T.D.N. Bovin & 76,37 & 79,63 & 80,82 & 79,61 & 73,37 & 79,45 \\
\hline T.D.N. Porc & 79 & 80,83 & $\mathrm{B1}, 82$ & 80,71 & 74,19 & 80,36 \\
\hline U. F. Bornn & 1,03 & 1,08 & 1,09 & 1,08 & 0,97 & 1,07 \\
\hline U. F. Porc & 1,07 & 1.09 & 1,11 & 1,09 & 0,99 & 1,08 \\
\hline E. M. Porc & 3239 & 3314 & 3354 & 3309 & 3041 & 3294 \\
\hline E. M. Volaille & 3172 & 3279 & 3328 & 3275 & 3017 & 3273 \\
\hline
\end{tabular}

1. Haute Côte d'Iroire - 2. Basse Côte d'Iroire (Récolte de petite saison des pluies) - 3. Moyenne Cotte d'Iroire - 4. Dahomey - 5. Bas Dahomey - 6. Togo

à la longueur variable du cycle végétatıf de la plante, au facteur variétal, mais il semble bien que la cause prépondérante soit climatique : les zones sèches et chaudes donnent beaucoup plus de grains cornés, plus riches en matières protéıques.

Les mêmes constatations ont été faites avec les sorghos et les petits mils; et les taux de matières protétques que l'on peut lire dans les tableaux I et II, sont déjà significatifs. Si les différences sont un peu moins nettes que pour les maïs, c'est que ceux-ci sont surtout cultivés dans des zones beaucoup plus humides.

Nous avons pu observer également que les mals des zones humides étaient souvent plus blancs que les mals des zones sèches. Les populations humaines qui consomment beaucoup de mais préfèrent les grains blancs pour leurs qualités culinaires. Mais pour l'alimentation anmale, nous tiendrons compte qu'ils sont, comme les autres grains, peu riches en vitamine ef provitamine $A$, alors que les grans jaunes ou orangés peuvent en contenır 30 à 40 fois plus.

Pour le reste, les mais de l'Ouest africain ne se distinguent pas des mais des zones tempérées. On a dif qu'ils étaient plus riches en matières grasses, plus cellulosıques, ce qui ne paraît pas en tout cas dans les résultats d'analyse que nous avons observés. Les teneurs en calcium et en phosphore sont également comparables, ainsi que le rapporf entre ces deux corps. Les taux de calcium sont, dans certains cas, nettement plus élevés que les taux compris entre 0,01 et 0,02 indiqués aux Etats-Unis ou en Europe. 
Enfin, les valeurs énergétiques sont, elles aussi, semblables à celles observées dans les pays à climat tempéré.

\section{Sons ef rofle de maiss (Tableau V).}

En colonne 1, on trouvera l'analyse d'un produit industriel, en colonne 2, celle d'un produit artisanal. Les sons de cette dernière catégorie sont, de loin, les plus fréquents en Afrique de l'Ouest. Les moulins artisanaux, mal réglés ou mal entretenus, de même que le broyage familial, laissent un pourcentage de sous-prodults très élevé, allant jusqu'à 45 p. 100 . Plus ce pourcentage est élevé, plus la composition du résıdu se rapproche de la composition de la céréale de base. Ce sont surtout les teneurs en cellutose ef en matières grasses, peu variables dans le grain entier, qui renseignent sur le taux de blutage; peu élevées, elles indıquent une mouture mal faite, avec matières résiduelies en quantités importantes.

Le produit de la colonne 1 est, à coup sûr, industriel par les taux élevés de cellulose et de matières grasses qu'il contient.

La difficulté de les conserver est le défaut majeur des sons de mais. En pays humide (Basse Côte-d'lvoire, Bas Dahomey) ils commencent à s'échauffer notablement $24 \mathrm{~h}$ après leur fabrication.

En colonne 3, nous avons donné la composition d'un épi de mais blanc (grain + rafle) broyé au concasseur à marteaux. Cette forme de présentation peut être intéressante, pour des

TABLEAU NOV

Sons et rafle de maīs

\begin{tabular}{|c|c|c|c|c|}
\hline & 1 & 2 & 3 & 4 \\
\hline Humidité & 13,53 & 13,99 & 6,04 & 10,88 \\
\hline Matière sèche & 86,47 & 86,01 & 93,96 & 89,12 \\
\hline M.P.H. & 10,93 & 11,90 & 7,49 & 1,65 \\
\hline Cellulose & 12,00 & 7,75 & 12,44 & 40,32 \\
\hline Mat. Erasses & 7,18 & 6,40 & 2,80 & 0,14 \\
\hline Mat. min. & 2,85 & 6,58 & 1,35 & 1,14 \\
\hline Calcium & 0,032 & 0,106 & 0,015 & 0,011 \\
\hline Fhosphore & 0,512 & 0,946 & 0,221 & 0,047 \\
\hline Ins. chl. & 0,18 & 2,09 & 0,14 & 0,23 \\
\hline E.N.A. & 53,51 & 53,38 & 63,31 & 45,78 \\
\hline M.P.D. Bovin & 6,33 & 6,90 & 5,45 & $0,3 i$ \\
\hline M.P.D. Porc & 8,30 & 9,04 & 5,40 & \\
\hline M.P.D. Volaille & 7,21 & 7,85 & & \\
\hline T.D.N. Bovin & 71,13 & 67,20 & 68,90 & 46,27 \\
\hline T.D.N. Pore & $66,80 ?$ & $64,97 ?$ & 65,14 & \\
\hline U. F. Bovin & 0,92 & 0,85 & 0,84 & 0,43 \\
\hline J. F. Porc & $0,85 ?$ & $0,82 ?$ & 0,80 & \\
\hline E. M. Parc & $2739 ?$ & $2663 ?$ & 2670 & \\
\hline E. M. Volaille & 1690 & 1744 & & \\
\hline
\end{tabular}

1. Son de mals Moulins Sentenac (Dakar) - 2. Son de maīs Katiola (Cote d'Ivoire) - 3. Epi de mars (grain + rafle) - 4. Rafle 
raısons d'économie ; les jeunes bovins, les bœufs de labour, les vaches laitières tirent un maximum de ce produit dans lequel les grains proprement dits représentent 72 p. 100 du poids et la rafle 28 p. 100. L'utılisation de ce produit est possible pour le porc.

La composition de la rafle seule, est donnée en colonne 4 ; elle vaut pour les bovins, et en ce qui concerne l'énergie, un bon foin de prairie : d'où utilisation éventuelle pour des boufs de labour.

Riz et dérivés (Tableau VI, VII, VIII).

Riz Paddy: C'est la graine obtenue par le cultivateur après «battage» du panicule. Le terme de «battage» a dans ce cas, une signification différente du battage du blé, opération qui sépare le caryopse de ses enveloppes.
Riz Corgo ou riz décortiqué : C'est le grain (caryopse) obtenu lorsque le paddy a été débarrassé de ses enveloppes pailieuses. Cette opération constifue le décorticage : elle se fait dans un appareil appelé décortiqueur dont il existe plusieurs types.

Balles: Ce sont les enveloppes qui recouvrent le caryopse.

Sons : Mélange de petites brisures arrachées au caryopse, de fines particules de balies, de germes et d'embryons détachés lors du décortıcage. On leur donne également le nom de « farines basses de riz cargo ».

Riz blanc: Le riz cargo passe dans des apparells appelés « cônes à blanchir » qui lui arrachent le péricarpe, les téguments séminaux

TABLEAU NO VI

Riz et dérivés

\begin{tabular}{|c|c|c|c|c|c|c|}
\hline & 1 & 2 & 3 & 4 & 5 & 6 \\
\hline Bumidité & 10,51 & 8,43 & 9,97 & 11,81 & 11,96 & 4,82 \\
\hline Matière sèche & 89,49 & 91.57 & 90,03 & 88,19 & 88,04 & 95,18 \\
\hline M.P.B. & 7,45 & 2,80 & 14,03 & 7,31 & 8,05 & 4,02 \\
\hline Celluiose & 8,42 & 33,25 & 8,09 & 0,18 & 0,70 & 33,50 \\
\hline Mat. Eragses & 2,13 & 0,00 & 12,04 & 0,67 & 1,32 & 1,10 \\
\hline Mat. $\min$. & 7,34 & 11,50 & 8,94 & 0,64 & 1,01 & 20,10 \\
\hline Calcium & 0,045 & 0,050 & 0,038 & 0,018 & 0,017 & 0,225 \\
\hline Phosphore & 0,280 & 0,046 & 1,482 & 0,126 & 0,180 & 0,078 \\
\hline Ins, chl. & 5,86 & 10,80 & 1,84 & 0,08 & 0,25 & 14,10 \\
\hline E.N.A. & 64,15 & 42,92 & 46,92 & 79,39 & 76,96 & 36,46 \\
\hline M.P.D. Bovin & - & 0,06 & 9,25 & - & - & 0,08 \\
\hline M.P.D. Pore & - & - & 12,20 & 6,41 & 6,92 & - \\
\hline M.P.D. Voleille & 5,21 & $=$ & 8,41 & 5,48 & 6,03 & - \\
\hline T.D.N. Bovin & - & 45,19 & 74,45 & - & - & 42,88 \\
\hline T.D.N, Pore & - & - & 83.25 & 86,75 & 86,03 & - \\
\hline D. F. Bovin & - & 0,39 & 0,96 & - & - & 0.32 \\
\hline U. F. Pore & - & - & 1,12 & 1,20 & 1,18 & - \\
\hline E. M. Porc & - & - & 3413 & 3557 & 3527 & - \\
\hline E. M. Volaille & 2630 & - & 2330 & 3057 & 3036 & \\
\hline
\end{tabular}

1. Riz pady Korhogo (Cote d'Ivoire) - 2. Halles du riz 1 - 3. Farine de cones du riz 1 - 4. Riz blanc de 1 5. Brisure de 1 - 6. Paille de riz de Korhogo (prélevée en pleine saison sèche). 
et la couche à aleurone. On obtient ainsi le riz blanc.

Farines de cônes: Ce sont les sous-produits enlevés au riz cargo par les « cônes à blanchir \%. On les appelle aussi «farines basses de blanchiment $\%$.

Brisures de riz blanc: A la sortie de l'appareil à décortiquer, les balles, sons, grans de riz cargo, etc..., sont mélangés. Des appareils annexes les séparent plus ou moins complètement. Mals, dans certains cas, les balles sont rejetées avec les sons, lorsque ceux-cl sont produits en trop petites quantités. Au cours de l'opération du blanchiment, certains grains de riz blanc se fragmentent en produisant des brisures. A la sortie des «cônes à blanchir», des appareils séparent les grains de riz blanc entiers et les brisures suivant leur calibre.

La définition exacte du produit que l'on utilise et la connaissance de la technologie appliquée permettent de juger de sa valeur bromatologique avec suffisamment de précision pour les calculs habituels de rationnement. En effet, chacun des produits et sous-produits de la rizerie a une composition assez spécifique, définie entre des limites voisines qui ne se recouvrent pas l'une et l'autre. L'exemple des tableaux VI, VII et VIII, va nous permettre de placer les principales issues du riz dans leur «contexte bromatologique $\gg$.

Les Balles : La colonne 4 du tablecu VIII, donne la composition moyenne d'une quarantaine

TABLEAU NO VII

Farines de cônes à blanchır

\begin{tabular}{|c|c|c|c|c|c|c|}
\hline & 1 & 2 & 3 & 4 & 5 & 6 \\
\hline Humidité & 8,08 & 7,60 & 11,13 & 9,63 & 9,83 & 15,00 \\
\hline Matière sèche & 91,92 & 92,40 & 88,87 & 90,37 & 90,17 & 85,00 \\
\hline M.P.B. & 8,73 & 10,78 & 11,58 & 11,25 & 11,37 & 13,38 \\
\hline Cellulose & 4,85 & 7,90 & 6,60 & 7,05 & 6,90 & 5,78 \\
\hline Mat. Erasaes & 8,34 & 10,90 & 10,44 & 15,63 & 15,67 & 14,40 \\
\hline Nat. min. & 4,57 & 10,28 & 5,76 & 6,04 & 6,15 & 7,56 \\
\hline Calciun & 0,046 & 0,057 & 0,039 & 0,060 & 0,070 & 0,051 \\
\hline Phosphore & 0,736 & 1,552 & 1,150 & 1,184 & 1,280 & 1,657 \\
\hline Ins, chl. & 1,33 & 2,79 & 0,66 & 0,65 & 0,70 & \\
\hline E.N.A. & 65,43 & 52,54 & 54,49 & 50,40 & 50,09 & 43,88 \\
\hline M.P.D. Bovin & 5,76 & 7,01 & 7,53 & 7,31 & 7,39 & 8,70 \\
\hline M.P.D. Porc & 7,60 & 9,38 & 10,07 & 5.79 & 9,89 & 11,64 \\
\hline M.P.D. Volaille & 5,24 & 6,46 & 6,95 & 6,75 & 6,82 & $B, 03$ \\
\hline T.D.N. Bovin & 81,38 & 75,48 & 70,69 & 81,36 & 81,19 & 74,39 \\
\hline T.D.N. Porc & 86,56 & 83,08 & 84,04 & 90,76 & 90,51 & 83,89 \\
\hline U. F. Bovin & 1,09 & 0,97 & 0,90 & 1,10 & 1,09 & 0,99 \\
\hline U. F. Porc & 1,21 & 1,11 & 1,15 & 1,26 & 1,25 & 1,15 \\
\hline E. M. Porc & 3540 & 3406 & 3446 & 3721 & 3710 & 3423 \\
\hline E. M. Volaille & 2343 & 2285 & 2309 & 2633 & 2623 & 2473 \\
\hline
\end{tabular}

1. Rizerie de Molodo (Mali) - 2. Rizerie de Diafarabe (Mali) - 3. Rizerıe de Richara-Toll (Sénégal) 4. Sénégal Issue. ler Cơne - 5. Sénégel Issue, 2ème Cône - 6. Rizerie de Korhogo (Cote d'Ivoire) 
d'échantillons de balles de riz, tirée de FEEDS and FEEDING. La colonne 3 donne la composition d'une balle prélevée à Dakar et originaıre des rizeries de RICHARD-TOLL. Les taux des principes alimentaires sont tout à fait comparables. Nous avons recherché le taux de l'insoluble chlorhydrique; il est très élevé sur toutes les balles (cutour de 18 p. 100), ce qui est un facteur très défavorable dans l'alimentation des animaux domestiques.

Les balles de riz peuvent-elles être données aux ruminants? C'est une question qui est souvent posée aux techniciens et à laquelie, on doit répondre négativement. Le T.D.N., compris entre 9 et 10, peut faire croire qu'il y a tout de même quelque chose à exploiter dans cet aliment pour un ruminant. Mais le calcul de la valeur fourragère exprimée en U.F. donne un chiffre négatif, ce qui signifie que l'énergie dépensée pour la digestion des balles est plus importante que l'énergie récupérable par l'organisme après catabolisme des principes absorbés. Les balles peuvent éventuellement être additionnées à d'autres matières plus substantielles; mais ce sera toujours en petite proportion car il faut craindre l'action de certains éléments de la balle qui pourralent abaisser les taux de digestibilité des autres aliments de la ration, la balle se comportant alors comme un agent de désassimilation et un « anti-aliment».

Les balles sont utılisées pour falsifier les sons de riz et même les farines de cônes à blanchir.

Les sons : La colonne 1 du tableau VIII, donne la composition d'un son de riz. Ces produits sont

TABLEAU NT VIII

Riz - sous produits divers

\begin{tabular}{|c|c|c|c|c|c|}
\hline & 1 & 2 & 3 & 4 & 5 \\
\hline Fumidité & 11,62 & 9,02 & 9,63 & 8 & 9,23 \\
\hline Matière sèche & 88,38 & 90,98 & 90,37 & 92 & 90.77 \\
\hline $\mathrm{M} . \mathrm{P} \cdot \mathrm{B}$ & 7,68 & 11,76 & 3,50 & 3 & 7,98 \\
\hline Cellulose & 19,90 & 9,45 & 38,20 & 40,7 & 1,05 \\
\hline Mat. Eresses & 3,31 & 14,31 & 1,09 & 0,80 & 0,89 \\
\hline Mat. min. & 7,15 & 9,45 & 19,05 & 19,1 & 1,35 \\
\hline Calcium & 0,082 & 0,032 & 0,08 & 0,08 & 0,032 \\
\hline Phosphore & 0,473 & 1,268 & 0,121 & 0,08 & 0,270 \\
\hline Ins. ohl. & 4,65 & 2,95 & 17,60 & & 0,25 \\
\hline E.N.A. & 50,34 & 46,01 & 28,53 & 28,4 & 79,50 \\
\hline M.P.D. Bovin & 4,99 & 7,64 & 0,14 & 0,12 & 5,27 \\
\hline M.P.D. Porc & 5,83 & 8,93 & & & 6,87 \\
\hline M.P.D. Volaille & & 7,76 & & & 5,98 \\
\hline T.D.N. Bovin & 51,00 & 70,62 & 9,50 & 9,90 & 80,90 \\
\hline T.D.N. Porc & 57,00 & 75,79 & & & 87,50 \\
\hline U. F. Sovin & 0,52 & 0.89 & & & 1,09 \\
\hline U. F. Pore & 0,67 & 0.99 & & & 1,22 \\
\hline E. M. Porc & 2337 & 3107 & & & 4009 \\
\hline E. M. Volaflle & & 1653 ? & & & 3096 \\
\hline
\end{tabular}

1. Son de riz Bougké (Cote d'Ivoire) - 2. Issue non précisée (Bobo-Dioulasso) - 3. Balle de riz - Dakar (Sénégal) - 4. Balle de riz tirée de "Feeds and Feeding" de Horrisson - 5. Issue dénommée criblure-rizerie de Miono (MA11) 
$\mathrm{d}_{\mathrm{e}}$ composition assez variable, mais ils sont caractérisés par un taux de cellulose assez élevé (de 13 d̀ 20 p. 100) ; le taux de matières grasses $y$ est toujours inférieur à 6 p. 100 . Certaıns appareils de décorticage (à rouleaux de caoutchouc en particulıer) donnent de très petites proportions de sons, moins de 4 p. 100 sur paddy: dans ce cas, on ne récupère pas ces sous-produits qui sont élimınés avec les balles. C'est le cas de la rizerie près de laquelie nous avons prélevé les échantillons énumérés au tableau VI.

S'ils contiennent trop de cellulose, ce qui est le cas lorsqu'à la sortie des décortiqueurs la séparation des balles est mal faite, les sons de riz ne peuvent être donnés qu'aux bovins pour lesquels ils constituent de bons aliments. Lorsque le pourcentage de celiulose n'est pas trop élevé, c'est le porc qui exploite le mieux ce prodult.

Les formes basses de riz ou farines de cônes d blanchir.

Le tableau VII, donne les résultats d'une série d'analyses d'échantillons d'origines différentes. Comparées aux sons et aux farınes qu'on pourrait éventuellement obtenir à partir des brisures, ces matières se distınguent par une teneur en cellulose intermédiaıre, toujours ınférieure d̀ 8 p. 100 ; un pourcentage en M. P. B. supérieur (par suite de la présence des cellules à aleurone). Elles sont caractérisées par une teneur quelquefois très élevée en $M . G$., surtout si les riz ont été étuvés avant le décortıcage : ce qu a sans doute été le cas pour les farines des colonnes 4 et 5 dont nous n'avons pu connaitre l'origine. La richesse en M. G. constitue un défaut pour le stockage prolongé de ces farines, mais ce n'est pas un vice rédhibitore pour leur emploi en alimentation anımale; elles sont de très bons produits pour le porc ef le poulet.

\section{Détermınation de la nature d'un produit de rizerie suivant sa composition.}

Tableau VI - colonne 2 : Balles du riz 1 - sa valeur alimentaire est nettement supérieure à celle que l'on attribue habituellement à ces produits (tableau VIII). En particulier, la teneur en insoluble chlorhydrique est beaucoup plus basse et le taux d'E. N. A. plus élevé. Dans cette rizerie, en effet, le son n'est pas récupéré à la sortie des décortiqueurs et est rejeté avec les balles. Dans ce cas, le produit vaut une bonne paille.

Tobleau VIII - colonne 2 : Issue non précisée échantillon reçu au Laboratoire sous la dénomination d'issue de riz. II s'agit d'une farine de cône à blanchir obtenue très probablement à partir d'un riz étuvé comme semble l'indiquer la haute teneur en matières grasses.

Tableau VIII - colonne 5 : Issue dénommée criblure - Nous avons prélevé ce sous-produit à la sortie d'un tarare installé après le décortiqueur. La composition montre qu'il ne s'agit ni d'un son, ni d'une balle. Elle se rapproche beaucoup d'un riz blanc qui aurait un taux de cellulose un peu élevé.

En fait, certaines rizeries ont après le décortiqueur une sérle d'appareıls à trier et de tarares qui séparent les produits bien différenciés sortant mélangés du décortiqueur. C'es $\dagger$ ce que nous avons trouvé à la rizerie de Niono (Mali), et l'échantıllon prélevé est une brisure de riz cargo (opposable aux brisures de riz blanc qui sorient des cônes à blanchir). Dans la plupart des cas, ces brisures entrent dans la composition des sons.

\section{Fonio (Tablequ IX).}

Digitaria Exilis; céréale dont le grain est très utilisé dans certaines régions de l'Ouest africain pour l'alimentation humaine.

Le fonio peut être intéressant à donner localement aux anımaux dans les régions de production; il ne fait pas l'objet d'échanges commerciaux Importants et c'est un produit cher lorsqu'il est acheté dans les pays non-producteurs.

L'intérêt de ce grain, au point de vue nutritionnel, est marqué par sa haute teneur en méthionine, tout à fait inhabituelle chez les céréales. CARBENIER, JAEGER, BUSSON, ont donné dans les «Annales de la Nutrition et de l'Alimentation - Tome XIV (1960) », le taux de 5,6 p. 100 de la matière protérque. Nous avons obtenu 5,9 p. 100 , valeur très voisine de la précédente. Les autres grains ont, dans la plupart des cas, un taux de méthıonine compris entre 1 et 2 p. 100 de la matière protéique. Par ailleurs, on sait que les aliments pour le bétail originaires des pays de l'Afrique de l'Ouest sont tous très déficients en cet acıde aminé. 
TABLEAU NO IX

Fonio

\begin{tabular}{|c|c|c|c|}
\hline & 1 & 2 & 3 \\
\hline Humidité & 10,50 & 11,4 & 10,2 \\
\hline Matière sèche & 89,40 & 88,6 & 89,8 \\
\hline M.P.B. & 9.03 & 6,6 & 6,7 \\
\hline Cellulose & 7,60 & 6,35 & 7,3 \\
\hline Mat. ETagaes & 2,23 & 2,95 & 2,6 \\
\hline Nat. min. & 12,96 & 9,00 & 14,75 \\
\hline Calcium & 0,192 & 0,068 & 0,197 \\
\hline Phosphore & 0,256 & 0,28 & 0,29 \\
\hline Ins. chl. & 9,07 & & \\
\hline E.N.A. & 56,98 & 63,7 & 58,45 \\
\hline M.P.D. Borin & & & \\
\hline M.P.D. Pore & & & \\
\hline M.P.D. Volaille & $7,37 ?$ & $4,95 ?$ & \\
\hline T.D.N. Bovin & & & \\
\hline T.D.N. Forc & & & \\
\hline U. F. Bovin & & & \\
\hline U. F. Porc & & & \\
\hline E. H. Porc & & & \\
\hline E. 1. Volatlle & $2540 ?$ & $2760 ?$ & \\
\hline
\end{tabular}

1. Acheté au marché d'Abidjan - 2. Origlnsire d'Odtenne (Cote d'Iroire) méthionine totale: 0,39 soit 5,9 pour cent de la matière protéique 3. Grain de même orlgine, pegsé au broyeur a marm tealux - grille de $1 \mathrm{~mm}$.

On notera la forte teneur en insoluble chlorhydrique du prodult de la colonne 1, et la forte teneur en matière mınérale de celui de la colonne 3 qui traduit certainement un taux élevé de silice. C'est la mauvaise technique de la récolte et du battage qui est responsable de la souillure du grain par de petites pierres, du sable, de la terre.

Le calcul de l'énergie pour les volailles a été effectué en assimılant le fonio à l'alpiste.

Les issues de blé (Tableaux $X, X I, X I I)$.

L'industrialisation de la meuneries'est effectuée ces dernıères années dons les pays de l'Afrique de l'Ouest.

Les issues de blé sont des matières intéressantes pour l'alimentation du bétail et peuvent entrer dans la composition de provendes dans des proportions quelquefoss importantes. Le fabricant d'aliments exige, pour ses matıères premières un approvisionnement régulıer en quantité et en qualité. L'industrie permet souvent de le satisfaire mals, en ce qui concerne les issues de ta meunerie, il faut connaître, avant tout, la correspondance entre les appellations et la composition des denrées, et vérifier si cette composition est constante, relativement à la terminologie aınsi définie.

Nous avons seulement retenu les termes de gros sons, sons fins et remoulages qui sont les plus usıtés dans l'atimentation anımale.

\section{Gros sons.}

Ce sont des issues essentiellement caractérisées par leur forte teneur en cellulose, ce qui interdit leur emploi à des taux élevés dans les concentrés de volailles. La teneur en M. P. B. est, en principe, comprise entre 8 et 11 p. 100.

L'examen du tableau $X$ montre que, sous la dénomination «gros sons de blé», on trouve des produits très variables quant à la teneur en cellulose et en M. P. B.

Les produits des 3 premières colonnes ne peuvent être donnés aux volaılles avec des résultats certaıns; les produits des colonnes 4 et 5 , moins riches en cellulose, peuvent être incorporés dans les rations poulettes et pondeuses à haut rendement.

\section{Les sons fins (Tabieau XI).}

Ces produits sont nettement plus riches en matières protérques que les gros sons; leur teneur en cellulose est normalement moins élevée, de telle sorte qu'ils peuvent être incorporés dans les alıments des volalles (hormis les poussins), et particulièrement des poulettes et des pondeuses.

En ce qui concerne la cellulose, les remarques que nous avons fates pour les gros sons s'appliquent aux sons fins, et le produit de la colonne 3 ne peut être préconısé pour les volailles.

On comparera surtout les produits des colonnes 1, 2 et 3 provenani, tous les tross, mass à 


\begin{tabular}{|c|c|c|c|c|c|}
\hline & 1 & 2 & 3 & 4 & 5 \\
\hline Hunidité & 14,44 & 11,80 & 11,00 & 7,62 & 11,49 \\
\hline Matière gèche & 85,56 & 88,20 & 89,00 & 92,38 & 88,51 \\
\hline$M_{\bullet} P_{\bullet} B_{\bullet}$ & 8,57 & 8,05 & 9,20 & 15,63 & 14,52 \\
\hline Celluziose & 15,30 & 16,95 & 17,20 & 13,80 & 10,40 \\
\hline Mat. Erasses & 1.96 & 1,35 & 3,13 & 3,05 & 2,56 \\
\hline 伤. min. & 5,30 & 4,1 & 4,57 & 7,09 & 5,96 \\
\hline Calcium & 0,210 & 0,15 & 0,088 & 0,126 & 0,102 \\
\hline Phosphore & 0,770 & 0,73 & 0,904 & 1,520 & 1,286 \\
\hline Ins. chl. & 0,25 & & 0,10 & 0,05 & 0,02 \\
\hline E.N.A. & 54,43 & 57,75 & 54,90 & 52,81 & 55,07 \\
\hline M.P.D. Bovin & 6,60 & 6,20 & 7,08 & 12,04 & 11,18 \\
\hline M.P.D. Forc & 6,51 & 6,12 & 6,99 & 11,58 & 11,03 \\
\hline M.P.D. Volaille & $5,65 ?$ & $5,31 ?$ & $6,07 ?$ & 10,31 & 9.58 \\
\hline T.D.N. Bovin & 69,41 & 61,20 & 63,54 & 65,04 & 60,48 \\
\hline$T_{0} D_{a} H_{.}$Porc & 50,26 & 51,68 & 52,91 & 55,47 & 54,77 \\
\hline D. F. Bovin & 0,73 & 0.72 & 0,76 & 0,77 & 0,70 \\
\hline D. F. Porc & 0,56 & 0,58 & 0,60 & 0,63 & 0,64 \\
\hline E. H. Porc & 2060 & 2188 & 2169 & 2274 & 2246 \\
\hline E. M. Volaille & $1395 ?$ & $1428 ?$ & $1472 ?$ & 1584 & 1575 \\
\hline
\end{tabular}

1. Grands morlins d'Abidjan (1962) - 2, grands moulins d'Abidjen (1963) - 3. grands moulins d'Abridjan (1964) 4. moulins de Niamey - 5. Moulins Sentenac (Dakar)

des époques différentes, des grands moulins d'Abidjan ; la règle de constance dans la composition que nous avons présentée comme nécessaire pour le fabricant d'aliments du bétail n'a pas été respectée. Celui-cl ne pourra plus incorporer le produit 3 dans des concentrés « poulets de chair» par exemple; il devra revoir ses formules et ses approvisionnements en matières premıères, ce qui est une gêne considérable.

Les remouloges (Tableau XII).

La teneur en cellulose de ces produits ne devrait pas excéder 8 p. 100. Ils sont un peu plus riches en matıères protéiques que les sons fins. Toutefois, cette dernière constatation ne doit pas être considérée comme un critère et c'est la teneur en cellulose qui doit surtout permettre de reconnaître un son fin d'un remoulage.

Nous avons été intrigués par les résultats des analyses consignés en colonne 6 (produit prélevé quelques semaines plus tard aux grands moulıns d'Abidjan). Ce remoulage ressemblait singulièrement à un son fin. L'administration de l'usine confirma que le produit était un mélange des trois issues vendu sous le nom et au prix du remoulage, comprenant environ $1 / 6$ de gros son, $1 / 6$ de son fin et 2/3 de remoulage.

Les remoulages peuvent être comparés, par leur origine, aux farines de cônes à blanchir le riz. Ces sous-produits proviennent de la partie périphérique du caryopse lorsque les sons ont été enlevés. Les remoulages ont des teneurs en 
TRBLFAO NO XI

Sons fins de blé

\begin{tabular}{|c|c|c|c|c|c|}
\hline & 1 & 2 & 3 & 4 & -5 \\
\hline Humidité & 14,33 & 12,55 & 10,93 & 7,81 & 13.41 \\
\hline Matière sèche & 85,67 & 87,45 & 89,07 & 92,19 & 86,59 \\
\hline M.P.E. & 13,82 & 15,30 & 12,40 & 16,82 & 15,41 \\
\hline Cellulose & 8,30 & 7,85 & 14,20 & 10,15 & 9,55 \\
\hline Mnt. grasses & 2,70 & 3,75 & 3,78 & 4,30 & 3,05 \\
\hline Mat. min. & 4,74 & 4,90 & 5,93 & 5,08 & 4,44 \\
\hline Caloium & 0,10 & 0,11 & 0,112 & 0,130 & 0,104 \\
\hline Phosphore & 0,90 & 1,15 & 1,200 & 1,080 & 0,976 \\
\hline Ins. chl. & 0,02 & & 0,12 & 0,05 & 0,06 \\
\hline E.N.A. & 56,11 & 55,65 & 52,76 & 55,84 & 54,14 \\
\hline M.P.D. Bovin & 10,23 & 11,32 & 10,78 & 12,44 & 11,40 \\
\hline M.P.D. Pore & 10,50 & 11,62 & 10,64 & 12,78 & 11,71 \\
\hline M.P.D. Volaille & 9,12 & 10,10 & 8,18 & 11,10 & 10,17 \\
\hline T.D.N. Bovin & 60,32 & 62,70 & 64,15 & 65,63 & 60,93 \\
\hline T.D.N. Porc & 56,00 & 56,70 & 54,02 & 59,26 & 55,25 \\
\hline J. F. Bovin & 0,71 & 0,75 & 0,77 & 0,78 & 0,72 \\
\hline D. F. Porc & 0,67 & 0,67 & 0,62 & 0,70 & 0,65 \\
\hline B. N. Pore & 2296 & 2324 & 2215 & 2430 & 2265 \\
\hline D. M. Volaille & 1579 & 1648 & 1531 & 1716 & 1597 \\
\hline
\end{tabular}

1. Grands mouling d'Abidjan (1962) - 2. Grands mouling d'Abidjan (1963) - 3. Grands mouljng d'Abidjan (1964) -

4. Moulins de Niamey - 5. Moulins Sentenac (Dakar)

M. P. B. plus élevées et les forines basses de rız des teneurs en M. G. supérieures. Les taux de cellulose sont comparables.

La valeur énergétique des farines de cônes dépasse la valeur énergétique des remoulages et Il est à remarquer que le porc est l'espèce qui transforme le mieux les premières et que ce sont les bovins adultes qui exploitent au maximum les seconds.

\section{Sous-produits de la fabrication industrielle de la bière}

Les Touraillons : Les graıns d'orge germés sont débarrassés de leurs radicelles ; celles-ci constituent les touraillons, le reste du grain forme le malt.
Les Drèches: Ce sont les résidus solıdes que I'on trouve au fond des cuves de brassage après la saccharification de l'amidon du malt. Les produits liquides forment le moût.

Les Levures: Le moût subit la fermentation après ensemencement par des levures. Une partie de celles-ci est récupérée après filtration.

Les brasseries industrielles de l'Ouest Africain (Dakar, Abidjan. Cotonou) reçoivent le malt préparé en Europe; Il n'y a donc pas de touraillons.

Dans les cuves de brassage, on ajoute des grains de production locale : gruaux de riz, de mais qui ont été cuits pour gélatiner l'amidon, ce qui peut donner des drèches de composition légèrement différente. 
Remoulages de blé tendre

\begin{tabular}{|c|c|c|c|c|c|c|}
\hline & 1 & 2 & 3 & 4 & 5 & 6 \\
\hline Elamidatté & 14,34 & 12,35 & 11.54 & 13,48 & 13,38 & 12,07 \\
\hline Matière sèche & 85,66 & 87,65 & 88,46 & 86,52 & 86,62 & 87,93 \\
\hline M.P.B. & 13,50 & $; 15,95$ & 15,30 & 16,78 & 17,04 & 15,47 \\
\hline Cellulose & 8,36 & 7,10 & 10,00 & 7,60 & 5,85 & 9,45 \\
\hline Mat. Erasges & 2,34 & 3,45 & 4,35 & 3,85 & 3,67 & 3,90 \\
\hline Mat. min. & 5,16 & 4,65 & 4.95 & 4,19 & 3,46 & 4,78 \\
\hline Calciun & 0,090 & 0,110 & 0,110 & 0,100 & 0,081 & 0,112 \\
\hline Phosphore & 0,710 & 1,100 & 1,012 & 0,916 & 0,776 & 1,032 \\
\hline Ins. chl. & 0,02 & & 0,09 & 0,04 & 0,03 & 0,10 \\
\hline E.N.A. & 56,30 & 56,50 & 53.86 & 54,10 & 56,60 & 54,33 \\
\hline M.P.D. Bovin & 11,20 & 13,24 & 11,63 & 13.95 & 15,00 & 11,76 \\
\hline M.P.D. Porc & 10,66 & 12,60 & 11,63 & 13,26 & 13,63 & 11,76 \\
\hline M.P.D. Volaille & 8,77 & 10,37 & 10,10 & 10,91 & 11,07 & 10,05 \\
\hline T.D.N. Bavin & 70,23 & 73,80 & 63,03 & 73,44 & 75,32 & 63,03 \\
\hline T.D.N. Porc & 57,83 & 61,50 & 56,74 & 61,12 & 67,68 & 57,00 \\
\hline U. F. Bovin & 0,90 & 0,96 & 0,75 & 0,95 & 1,00 & 0,75 \\
\hline U. F. Pore & 0,70 & 0,75 & 0,70 & 0,74 & 0,87 & 0,70 \\
\hline E. M. Forc & 2371 & 2521 & 2326 & 2505 & 2775 & 2337 \\
\hline $\mathrm{E}_{0}$ M. Volaille & 1797 & 1889 & 1641 & 1875 & 1890 & 1635 \\
\hline
\end{tabular}

1. Grands moulins d'Abidjan (1962) - 2. Grands mov2ins d'Abidjan (1963)- 3. Grands moulins d'Abidjan (1964) 4. Moulins Sentenac Dakar (1964) remoulages bis - 5. Mouling Sentenac Dakar (1964) remoulagea blancs -6 . Grends mơlins d'Abidjan (1964) (prélevés à la station de Bingerville)

Les résultats consignés au tableau XIII, portent sur des produits qui ont été séchés pour être expédiés au Laboratorre dans de bonnes conditions. La teneur en eau des drèches à la sortie de la brasserie, après quelques minutes d'égouttage, est de 70 d̀ 75 p. 100. La valeur bromatologique des produits que nous avons recueillis est comparable à la valeur de leurs homologues des pays européens, mais la teneur en $M$. P. B. est un peu plus élevée ainsi que la teneur en cellulose.

La comparaison des valeurs énergétıques pour les bovins d'une part et pour les porcs d'autre part, montre que les bovins utilisent beaucoup mieux les drèches de brasserie'; la différence est de l'ordre de 70 à 75 p. 100 par rapport aux valeurs les plus basses. C'est que le coefficient d'utilisation digestive est melleur chez les bovins; l'extractif non azoté des drèches contient des pentosanes dont l'absorption intestinale chez les monogastriques ef le porc en particulier, est très peu élevée.

On sait classiquement, que c'est à la vache laitière que convient le mieux cet alıment; on notera également qu'il a une action décalcifiante, cette action peut s'accentuer dans les pays chauds par suite de l'acidıté qui se développe rapidement dans le produit lorsqu'il n'est pas convenablement séché.

Les drèches industrielles séchées ont, pour les bovins, d'après MORRISSON, une valeur un peu supérieure aux sons de blé (Wheat Bran); cet auteur estime qu'en raison de leur encombre- 
TLATLEAD NO

Sous-produits de la brasserie industrielle

\begin{tabular}{|c|c|c|c|c|}
\hline & $t$ & 2 & 3 & 4 \\
\hline Humidité & 7,87 & 7,87 & 8,25 & 10,9 \\
\hline Matière sèche & 92,13 & 92,13 & 91,75 & 89,1 \\
\hline $\mathrm{H}_{\mathbf{*}} \mathrm{P}_{\mathbf{B}} \mathrm{B}_{\text {。 }}$ & 23,55 & 17,88 & 22,92 & 48,29 \\
\hline Cellulose & 22,17 & 17,36 & 19,80 & 0,88 \\
\hline rat. Erasseg & 6,31 & 5,59 & 5,43 & 0,55 \\
\hline Kat. min. & 2,96 & 3,64 & 3.53 & 8,80 \\
\hline Calcium & 0,157 & 0,193 & 0,220 & 0,088 \\
\hline Phosphore & 0,290 & 0,417 & 0,426 & 1.749 \\
\hline Ins, chl. & 0,16 & 1,54 & 1,37 & 0,33 \\
\hline $\mathrm{E}_{\Delta} \mathrm{H}_{4} \mathrm{~A}_{\bullet}$ & 37,17 & 47,62 & 40,07 & 30.58 \\
\hline M.P.D. Bovin & 17,18 & 13,05 & 16,73 & \\
\hline H.P.D. Pore & 18,60 & 14,10 & 18,10 & 42,46 \\
\hline H.P.D. Volaille & & & & 40,04 \\
\hline T.D.N. Bovin & 61,51 & 60,85 & 59,00 & \\
\hline T.D.N. Poro & 37,60 & 34,88 & 36,65 & 69,09 \\
\hline J. F. Bovin & 0,69 & 0,68 & 0,64 & \\
\hline U. F. Porc & 0,33 & 0,27 & 0,31 & 0,88 \\
\hline E. M. Porc & 1540 & 1431 & 1503 & 2794 \\
\hline E. M. Volaille & & & & 2486 \\
\hline
\end{tabular}

1. Drèche brasserie Bracodi (Abidjan) - 2. Dròche brasaerie Solibrá (Abidjan) - 3. Drèche brasserie OuestAfricain (Dakar) - 4. Levure brasserle Bracodi (Abidjan)

ment, les drèches ne sont pas un bon aliment pour les porcs, mais qu'elles peuvent remplacer l'avoine pour les truies nourrices.

\section{Sous-produit de la brasserie artisanale (Tableau XIV)}

La technique de la fabrication du «dolo» à partir des mils et sorghos et quelquefois du mals, peut être très élaborée (Haute-Volta par exemple). On y retrouve les phases principales des technıques industrielles: maltage, touraillage, brossage (saccharification) et fermentation.

Toutefois, les produits que nous avons recueillis présentent quelques différences de composition avec leurs homologues industriels; les matières premières ne sont pas les mêmes et les actions enzymatiques sont probablement un peu différentes.

On ne frouve habituellement qu'un seul sousproduit, celui dont l'analyse figure dans les colonnes 4,5 et 6 . C'est un mélange de touraillons (10 à 20 p. 100 ?), et de drèches. Ces tourallons sont en principe, les radicelles du mil ou du maîs germé, mais leur teneur en cellulose est nettement plus élevée que celle des produlis venant de l'orge malté. Cela vient de ce que, dans la fechnique artisanale. le graın malté est broyé avant le «touraillage», et que les germes sont séparés par flottaison et non par brossage ou arrachage comme dans la technique industrielle. En même temps que les germes, les 
Sous-produita de la brasserie artisangle

\begin{tabular}{|c|c|c|c|c|c|c|}
\hline & 1 & 2 & 3 & 4 & 5 & 6 \\
\hline Humidite & 8,70 & 10,20 & $.8,91$ & 5,17 & 7,9 & 8,95 \\
\hline Matière gèche & 91,30 & 89,80 & 91,09 & 94,83 & 92,1 & 91,05 \\
\hline M.P.B. & 9,84 & 24,90 & 27,06 & 22.75 & 20,7 & 14,70 \\
\hline Cellulose & 3,30 & 16,45 & 5,45 & 10,30 & 11,46 & 10,80 \\
\hline Mat. grasses & 1,38 & 8,10 & 11,25 & 5,53 & 6,51 & 6,42 \\
\hline Mat. min. & 1,79 & 9,74 & 4,26 & 3,47 & 10,95 & 3.09 \\
\hline Celciurr & 0,028 & 0,133 & 0,038 & & 0,030 & 0,030 \\
\hline Phosphore & 0,252 & 0,458 & 0,241 & & 0,357 & 0,240 \\
\hline Ins. chl. & 0,33 & 0,93 & 2,60 & . & 8,34 & 1.52 \\
\hline E.N.A. & 75 & 36,61 & 43,07 & 52,78 & 42,48 & 56,04 \\
\hline M.P.D. Bovin & 5,70 & $15,93 ?$ & 19.75 & 16,61 & 15,12 & 10,74 \\
\hline M.P.D. Porc & 7,58 & $17.43 ?$ & 21,38 & 17,97 & 16,35 & 11,61 \\
\hline M.P.D. Volaille & 8,26 & & & & & \\
\hline T.D.N. Bovin & 63,37 & $67.63 ?$ & 70,72 & 64.85 & 59,07 & 62,94 \\
\hline T.D.N. Pore & 83,71 & $38,58 ?$ & 48,30 & 39.90 & $36,84 \ldots$ & 35,64 \\
\hline J. F. Bovin & 0,75 & $0,84 ?$ & 0,89 & 0,76 & 0,66 & 0.75 \\
\hline U. F. Porc & 1,13 & $0,35 ?$ & 0,51 & 0,35 & 0,30 & 0,30 \\
\hline E. H. Porc & 3432 & $1583 ?$ & 1980 & 1636 & 1512 & $\uparrow 461$ \\
\hline E. M. Volaille & 3363 & & & & & \\
\hline
\end{tabular}

1. Sorgho rouge germe-Ouagadougou (Houte-Volta) - 2. Touraillons du sorgho 1 - 3. Drèches du sorgho 1 - 4. Drèches de sorgho-Bobodioulssso (Haute-Volta) - 5. Drêches de petit mil Bouaké (Cote d'Ivoire) - 6. Drtrchea de maIs Bouaké (Côte d'Iroire).

débris d'enveloppes, plus cellulosiques que le grain, viennent flotter à la surface de l'eau où ils sont recueillıs puis séchés pour donner le produit de la colonne 2. La drèche, au sens propre du terme est donnée colonne 3.

La technique artisanale, même très perfectionnée, est toujours moins él aborée que la technique industrielle; «l'épuisement des grains» en amidon, en partıculıer, est moıns poussé ; aussi, ces «drèches» sont-elles plus riches en E. N.A. et proportionnellement moins riches en cellulose.

La séparation des tourallons ef des drèches ne présente aucun intérêt au point de vue de l'alimentation des animaux, en Afrique, et c'est le mélange qui est toujours récupéré et donne un sous-praduit bon marché et de bonne valeur pour les ruminants.
On remarquera la teneur peu élevée en $M$. P. B. des drèches de mals. Bien que nous n'ayons recueilli qu'un échantilion de ce sous-produit, it semble que toutes les drèches de mais ont un taux moins élevé en ce principe que les drèches de mil ; les bières de maïs seraient, en effet, plus riches en protides; le graın est donc davantage épuisé en ces principes.

Enfin, d'après les calculs que nous avons pu faire, à partir des renseignements obtenus au cours de notre enquête en Haulte-Volta, il faudrait 11 à $12 \mathrm{~kg}$ de sorgho pour faire 50 à 55 । de dolo: de plus les sous-produits de cette fabrication représentent 30 à 35 p. 100 environ de la quantité de mil employé, leur teneur en eau étant supposée égale à celle du grain employé au départ. 


\section{B. - AUTRES VÉGÉTAUX ESSENTIELLEMENT FARINEUX}

Le manioc (Centre de Rech. zoot. Minankro) (Tableau XV)

Dans cette station, des observations concernant le manioc sont relevées depuis quelques années ; elles ont eu, d'abord, comme objectif, l'étude agronomique de la culture et le prix de revient de la matière première. Actuellement, elles portent sur la valeur alimentaire ef l'utilisation en quantités importantes dans les ratıons des animaux domestiques y compris les volailles et les veaux.

Bien que cet article soit une étude des valeurs techniques des sous-produits, nous pensons utile de donner les conclusions de nos observations sur les prix du manioc. A Bouaké-
Minankro, le kilo de monioc frais, sur champ revient à 1,50 à $2 \mathrm{~F} \mathrm{C.F.A.} \mathrm{II} \mathrm{en} \mathrm{faut} 2 \mathrm{~kg} 1 / 2$ pour faire $1 \mathrm{~kg}$ de cossettes séchées. Ce qui met l'unité fourragère à $5 \mathrm{~F}$ pour le porc et moins de $6 \mathrm{~F}$ pour le bouf, en ne tenant compte que de la valeur de la matière : Il faut y ajouter les frais de transport et de séchage, variables avec les conditions et les lieux d'emplor. Des observations faites par ailleurs confirment que c'est le manioc sec qui fournit l'énergie au prix le plus bas dans les pays où sa culture est traditionnelle et où les conditions écologiques sont favorables (Côted'lvoire-Dahomey-Togo).

Nous pensons que, dans l'état actuel de l'élevage dans ces pays, c'est la seule plante dont on peut préconiser la culture pour l'alimentation animale.

TABLEAU $\mathrm{N}^{\circ} \mathrm{XV}$

Cossettes de manioc de Bouaké-Minankro (COte d'Ivoire)

\begin{tabular}{|c|c|c|c|c|c|c|}
\hline & 1 & 2 & 3 & 4 & 5 & 6 \\
\hline Humtait té & 11,05 & 12,55 & 8,78 & 8,60 & 12,65 & 12,00 \\
\hline Matlère sèche & 88,95 & 87,45 & 91,22 & 91,40 & 87,35 & 88,00 \\
\hline M.P.B. & 2,05 & 2,88 & 2,73 & 2,43 & 2,50 & 2,54 \\
\hline Cellulose & 2,82 & 4,00 & 3,40 & 3,08 & 1,62 & 2,23 \\
\hline Mat. grasses & 0,65 & 0,84 & 0,86 & 1,27 & 0,50 & 0,44 \\
\hline Mat. min. & 2,89 & 2,66 & 3,45 & 2,45 & 2,47 & 2,43 \\
\hline Calcium & 0,112 & 0,110 & 0,120 & 0,102 & 0,120 & 0,100 \\
\hline Phosphore & 0,104 & 0,091 & 0,140 & 0,085 & 0,078 & 0,077 \\
\hline Ins. chl. & 0,46 & 0,77 & 0,71 & 0,45 & 0,24 & 0,24 \\
\hline E.N.Á. & 80,54 & 77,07 & 80,36 & 81,77 & 82,38 & 80,36 \\
\hline M.P.D. Bovin & 0 & 0 & 0 & 0 & 0 & 0 \\
\hline M.P.D. Porc & 1,39 & 1,96 & 1,85 & 1,65 & 1.70 & 1,73 \\
\hline M.P.D. Volaille & & 2,16 & & & & \\
\hline T.D.N. Bovin & 74,8 & 71,52 & 74,57 & 75,88 & 76,44 & 74,57 \\
\hline T.D.N. Pore & 83,6 & 81,73 & 84,44 & 85,60 & 81,72 & 83,20 \\
\hline U. F. Bouln & 0,98 & 0,92 & 0,96 & 0,99 & 1,02 & 0,98 \\
\hline U. F. Pore & 1,14 & 1,11 & 1,12 & 1,16 & 1,16 & 1,13 \\
\hline E. M. Porc & 3428 & 3351 & 3460 & 3509 & 3460 & 3410 \\
\hline E. M. Volaille & & 3071 & & & & \\
\hline
\end{tabular}

1. Manioc de 18 mois - Acıde cyanhydrique : 33,7 mg par kilog de matière sèche. - 2. Farine de 1 - 3. Manioc de 30 mojs - 4. Manioc de 20 mois - 5. Kanioc de 20 mois - 6. Nanioc de 20 mois 


\section{Manioc frais et sec}

Le tableau XVI donne les compositions de 3 produits frais et 1 prodult séché.

L'observation essentielle, au point de vue bromatologique, concerne les valeurs énergétiques pour bovins. II faut $2,8 \mathrm{~kg}$ environ des produits 1,2 ef 3 pour faire $1 \mathrm{~kg}$ du produit sec $n^{\circ} 4$. La valeur énergétıque moyenne du manioc frais est de 0,18 U.F. ; $2,8 \mathrm{~kg}$ fournissent donc 0,50 U. F. ; or, le calcul donne pour le manioc sec correspondant $0,96 \cup$. F. (colonne 4) ! D'après MORRISSON, les coefficients de digestibilité du manioc frais pour les bovins sont beaucoup plus faibles que ceux du manioc sec, ce qui se tradult par une valeur énergétique plus falble du premier produit, toutes choses étant égales par gilleurs.

Nous n'avons pu, malheureusement, trouver les mêmes éléments de comparaison pour le porc: tous les chiffres que nous avons relevés dans les ouvrages spécialisés se rapportent au manioc séché el surtout à la farine de manioc; cela vient de ce que les essais de digestibilité ont été faits dans les pays à climats tempérés où le manioc est uniquement utilisé sous forme sèche.

Nous n'avons donc effectué aucun calcul de T.D. N., d'U.F., et d'E.M., pour le manioc frais chez le porc.

On notera la constance de la composition des maniocs frais orıginaires des pays côtiers, sauf en ce qui concerne l'acide cyanhydrique; les

TAELEAU NO XVI

Mentoc frais et sec

\begin{tabular}{|c|c|c|c|c|}
\hline & 1 & 2 & 3 & 4 \\
\hline Huridites & 67,25 & 64,30 & 65.70 & 7,94 \\
\hline Matière sèche & 32,75 & 35,70 & 34,30 & 92,06 \\
\hline A.P.B. & 1,37 & 1,02 & 1,34 & 5,00 \\
\hline Cellulose & 0,80 & 0,90 & 0,54 & 3,90 \\
\hline Mat. Grapes & 0,14 & 0,28 & 0,34 & 0,60 \\
\hline Mat, min. & 0,69 & 0,97 & 1,13 & 3,48 \\
\hline Celoium & 0,071 & 0,034 & 0,027 & 0,153 \\
\hline Phouphore & 0,048 & 0,079 & 0,02 & 0,117 \\
\hline Ins. obl. & 0,11 & 0,05 & & 0,29 \\
\hline E.T.1. & 29,75 & 32,53 & 30,95 & 79,08 \\
\hline M.P.D. Borln & 0 & o & 0 & 0 \\
\hline M.P.D, Porc & & & & 3.40 \\
\hline \multicolumn{5}{|l|}{ X.P.D. Volatille } \\
\hline T.D.Y. Borly & 17,81 & 19,55 & 18,87 & 75,00 \\
\hline T.D.I. Poro & & & & 84,96 \\
\hline 0. F. Booln & 0,17 & 0,19 & 0,18 & 0,96 \\
\hline U. F. Porc & & & & 1,15 \\
\hline E. M. Pone & & & & 3483 \\
\hline E. F. Volntile & & & & \\
\hline
\end{tabular}

1. Manloc frais - Bingervillo (cote d'Ivoire) acide cyaniydrique 1.710 mg par kllog de M.S. - 2. Marioc frais (Cote d'Iroire)-acide cyanhydrIque 28,7 mg par lolog de M.S. - 3. Manioc freis (Dahomey) - 4. Cossettes de Kanioc sec-Sotube (Mnli). 
chiffres que l'on trouvera aux tableaux $X V$ ef $X V I$, se rapportent à des produits non épluchés. Le manioc $n^{0} 1$ du tableau $X V I$ a été acheté au marché de Bıngerville! Le taux maxımum admissible est de $20 \mathrm{mg}$ par $\mathrm{kg}$ d'aliment concentré (renseıgnement obtenu auprès d'industriels français) ce qui, en fonction des formules moyennes utilisées, signifie qu'il ne faudrait pas que le manioc dose plus de $50 \mathrm{mg}$ d'acide cyanhydrique. Pour en abaisser considérablement le taux, il suffit, théorıquement, de l'éplucher convenablement.

\section{Autres racines ef fubercules}

Les valeurs alımentaires et les meilleures conditions d'emploi de ces produits sont mal connues, squf pour la patate douce très utılisée en ExtrêmeOrient.

Aussi, le tableau XVII est-Il incomplet.

En Afrique intertropicale, ils ne seront employés qu'exceptionnellement pour les animaux domestiques par suite de leur prix plus élevé que celui du manioc.

\section{Banane (Tableau XVili)}

Les renseignements précis, déterminés à la suite de recherches ou d'essais sur les coefficients de digestibilité de la banane à l'état frass ou en farine, sont très rares. Nous n'avons trouvé d'exemple que dans Feeds of the World (SCHNEIDER) pour les moutons et chèvres que nous avons assimilés aux bovins adultes.

TAHLEAU No NII

Lutres racines et tubercules

\begin{tabular}{|c|c|c|c|c|c|c|}
\hline & 1 & 2 & 3 & 4 & 5 & 6 \\
\hline Bumidité & 79,50 & 66,00 & 62,20 & 64,10 & 58,25 & 72,30 \\
\hline Wtière sèche & 21,50 & 34,00 & 37,80 & 35,90 & 41,75 & 27,70 \\
\hline M.P.B. & 1,31 & 1,02 & 1,48 & 2,53 & 2,94 & 1,81 \\
\hline Cellulose & 0,58 & 1,24 & 0,95 & 1,15 & 1,04 & 0,38 \\
\hline Mat. grasses & 0,77 & 0,60 & 0,12 & $0, \infty$ & 0,14 & \\
\hline Mat. min. & 0,17 & 1,30 & $1, \infty$ & 1,29 & 1,41 & 0,94 \\
\hline Calcium & 0,025 & 0,069 & 0,157 & 0,075 & 0,030 & 0,022 \\
\hline Phosphore & 0,047 & 0,064 & 0,051 & 0,054 & 0,122 & 0.055 \\
\hline Ine. chI. & & 0,12 & 0,08 & 0,08 & 0,20 & \\
\hline E.Nak. & 18,67 & 29,84 & 34,25 & 30,84 & 36,22 & 24,45 \\
\hline M.P.D. Bovin & 0,85 & 0,66 & & & & \\
\hline M.P.D. Paro & 0,67 & 0,52 & $1.01 ?$ & $1,72 ?$ & $1,50 ?$ & \\
\hline \multicolumn{7}{|l|}{ K.P.D. Volatlle } \\
\hline T.D.F. Borin & 15,80 & 23,60 & & & & \\
\hline T.D.D. Poro & 20,00 & 29,89 & $35.78 ?$ & $35,17 ?$ & $36,74 ?$ & \\
\hline O. F. Borin & 0,19 & 0,27 & & & & \\
\hline J. F. Porc & 0.27 & 0.40 & $0,49 ?$ & $0,45 ?$ & $0.49 ?$ & \\
\hline E. H. Porc & 820 & 1226 & 1464 & $1360 ?$ & $1506 ?$ & \\
\hline I. 1. Volaille & & & & & & \\
\hline
\end{tabular}

1. Patate douce -Bingerville (Coto d'Iroiro) - 2. Patate douce- marché de Treichrille (Cotte d'Iroire) -

3. I gname de 6 mois -Bingerpllle (Cote d'Ivoire) - 4. Igname de 12 mois -Bingerville (Cote diIvoirg) - 5. Taro Yhrohé de Treichrille (Cote d'Irodre) - 6. Taro -Bingerville (Cote d'Ivoire) 
TABLEAD N० XVIII

Banane

\begin{tabular}{|c|c|c|c|c|c|c|}
\hline & 1 & 2 & 3 & 4 & 5 & 6 \\
\hline Hunidité & 76,00 & 75,85 & 75,5 & 11,65 & 10,30 & 1130 \\
\hline Watière sèche & $24, \infty 0$ & 24,15 & 24,5 & 88,35 & 89,70 & 88,70 \\
\hline M.P.B. & 1,43 & 1,29 & 1,90 & 3,48 & 2,68 & 5,19 \\
\hline Cellulose & 1,15 & 0,45 & 3,20 & 2,49 & 1,82 & 3,94 \\
\hline Mat. Bragses & 0,32 & 0,10 & 0,90 & 0,84 & 0,35 & 1,87 \\
\hline Mat. min. & 1,24 & 0,76 & 2,70 & 3,30 & 2,53 & 4,94 \\
\hline Calcilum & 0,017 & 0,013 & 0.032 & 0,045 & 0,013 & 0,11 \\
\hline Fhosphore & 0,029 & 0,027 & 0,036 & 0,060 & 0,054 & 0,071 \\
\hline Ing, chl. & 0,06 & 0,02 & 0,15 & 0,09 & 0,06 & 0,15 \\
\hline E.N.1. & 19,86 & 21,55 & 15,64 & 78,24 & 83,32 & 67,57 \\
\hline M.P.D. Bovin & 0,78 & 0,71 & 1.04 & 0 & & 1,76 \\
\hline \multicolumn{7}{|l|}{ M.P.D. Porc } \\
\hline \multicolumn{7}{|l|}{ M.P.D. Volaille } \\
\hline T.D.N. Bovin & 18,70 & 16,68 & 17,38 & 68,50 & & 58,55 \\
\hline \multicolumn{7}{|l|}{ T.D.H. Porc } \\
\hline$U_{0} F_{*}$ Bovin & 0,23 & 0,25 & 0,21 & 0,85 & & 0.74 \\
\hline \multicolumn{7}{|l|}{ J. F'. Porc } \\
\hline \multicolumn{7}{|l|}{ E. M。 Porc } \\
\hline E. $M_{0}$ Volatile & & & & & & \\
\hline
\end{tabular}

1. Banane poyo verte entiere (Cote d'Iroire) - 2. Polpe de benane 1 - 3. Pelure de banane 1 - 4. Farina de benane plantain entière (Cote d'Ivolre) - 5. Farine de pulpe de banane $4-6$. Farine de pelure de banane 4

Pour les porcs, nous n'avons rien trouvé : ce qui est fâcheux, car la banane est souvent donnée à ces animaux, et des questions la concernant sont fréquemment posées aux techniciens.

DAUMAS, dans une éfude entreprise à Madagascar, a déterminé la valeur énergétique de la banane entière et de la farine de banane, pour les monogastriques.

Il a utilisé la méthode de JACQUOT et GUILLEMENT, basée sur le dosage de l'insoluble formique.

Les valeurs trouvées sont les suivantes:

banane entière fraîche (avec peau) :0,2 UF/ $\mathrm{kg}$, farine de banane entière $\quad 1 \mathrm{UF} / \mathrm{kg}$, cossette de banane $\quad 1,08 \mathrm{UF} / \mathrm{kg}$.

Par ailleurs, on a pensé à l'emploi de la farine de banane dans le sevrage précace des veaux: on sait que cette farine est utilisée en diététique infantile. L'amylase contenue dans le fruit favorise-t-elle, chez les jeunes mammifères, la digestion précose des amylopectines, constituants essentiels des amidons de la banane?

Des recherches concernant la valeur alimentaire de ce fruit pourraient peut-être déboucher sur l'utilisation rationnelle de surplus invendables dans les pays ou il y a une grosse production bananıère.

Légumineuses - graines ef feuilles (Tableau XIX)

Dans les colonnes 1,2 et 3 , on trouvera des analyses se rapportant aux Niébés, petits haricots du genre Vigna (en anglais, Cowpea). 


\begin{tabular}{|l|c|c|c|c|c|c|}
\hline & 1 & 2 & 3 & 4 & 5 & 6 \\
\hline Fumidilté & 8,83 & 6,47 & 5,69 & 6,26 & 14,70 & 7,07 \\
\hline Matière sèche & 91,17 & 93,53 & 94,31 & 93,74 & 85,30 & 92,93 \\
\hline M.P.B. & 25,81 & 21,44 & 22,68 & 17,93 & 20,00 & 16,46 \\
\hline Cellulose & 4,10 & 2,50 & 5,90 & 7,23 & 8,50 & 25,48 \\
\hline Mat. Erasses & 1,62 & 1,25 & 1,15 & 6,60 & 1,37 & 2,28 \\
\hline Mat. min. & 3,99 & 3,18 & 3,19 & 3,07 & 3,72 & 7,05 \\
\hline Calcium & 0,168 & 0,093 & 0,094 & 0,054 & 0,142 & \\
\hline Phosphore & 0,373 & 0,290 & 0,275 & 0,190 & 0,296 & \\
\hline Ins. ohl. & 0,25 & 0,06 & 0,07 & 0,15 & 0,13 & \\
\hline E.N.A. & 55,65 & 65,16 & 61,39 & 58,91 & 51,71 & 41,66 \\
\hline \hline M.P.D. Borin & 21,16 & 17,58 & 18,60 & & & \\
\hline M.P.D. Porc & 24,26 & 20,15 & 21,32 & & 18,40 & 7,57 \\
\hline M.P.D. Volaille & & & & & & 12,35 \\
\hline T.D.N. Bovin & 78,23 & 81,85 & 81,37 & & & \\
\hline T.D.N. Porc & 77,73 & 81,08 & 80,44 & & 71,29 & 33,44 \\
\hline U. F. Bovin & 1,03 & 1,09 & 1,08 & & & \\
\hline U. F. Porc & 1,02 & 1,07 & 1,06 & & 0,93 & 0,25 \\
\hline E. M. Porc & 3186 & 3324 & 3298 & & 2923 & 1371 \\
\hline E. M. Volaille & & & & & & 1110 \\
\hline
\end{tabular}

1. Niebe Sotuba (Mali) - 2. Niebe b]anc Maradi (Niger) - 3. Niebe rouge Maradi (Niger) - 4. Pois de terre Maradi (Niger) - 5. Pois d'angole-Boukes (Cote d'Ivoire) - 6. Tarine de feujlie de pois d'angole (Mali)

Dans la colonne 4, le Pois de terre ou Woandzou voondzera sub-terronea (Gooper Congo des Anglo-Saxons).

Enfin, en colonnes 5 et 6 , on trouvera les compositions des grains et des feuilles de Pois d'Angole - Cajanus Indicus (Pıgeon pea en anglaıs).

Nous n'avons trouvé aucun renseignement sur les digestıbılités de ces graines chez les volaılles. On constate leur bonne valeur alımentaire chez le porc; toutefois, ils ne doivent être utilisés qu'en compléments, pour supplémenter une ration. S'ils constituent la base de l'alimentation, ils peuvent provoquer des troubles digestifs. Leur qualité de supplémentation vient essentiellement de leurs protéines, très riches en lysine, acıde aminé déficient dans les aliments de
l'Afrique de l'Ouest. Mais on tiendra compte que les graines de légumineuses sont pauvres en acides aminés soufrés (méthionıne, cystıne).

\section{C. - LES gRAINES OLÉAGINEUSES ET LEURS SOUS-PRODUITS}

\section{Tourteoux d'arachides « expellers»}

(Tableau $X X$ ef $X X I 1)$

Les 10 tourteaux expellers, dont les compositions sont rapportées dans les tableaux $X X$ et $X X I$, peuvent être consıdérés dans leur ensemble, comme des produits de bonne ef même de très bonne qualité (tourteau $n^{0} 2$ du tableau $X \times 1$ ) au point de vue bromatologique. Les teneurs en 
TABLABAU N $\mathbb{N}^{\circ} X X$

Tourteaux d'arachide "expellers"

\begin{tabular}{|c|c|c|c|c|c|c|}
\hline & 1 & 2 & 3 & 4 & 5 & 6 \\
\hline Humidité & 9,53 & 9,02 & 9,60 & 11,90 & 7,80 & 7,79 \\
\hline 梅tière sèche & 90,47 & 90,98 & 90,40 & 88,10 & 92,20 & 92,21 \\
\hline M.P.B. & 49,07 & 44,31 & $4 t, 66$ & 43,94 & 47,93 & 43,01 \\
\hline Cellulose & 5,42 & 6,98 & 6,83 & 4,23 & 6,36 & 6,45 \\
\hline Mat. grasseg & 7,74 & 4,00 & 5,88 & 5,14 & 5,40 & 6,26 \\
\hline Mat. min. & 4,70 & 7,34 & 5,31 & 5.73 & 6,71 & 6,60 \\
\hline Calcium & 0,144 & 0,16 & 0,097 & 0,246 & 0,103 & 0,104 \\
\hline Phosphore & 0,687 & 0,49 & 0,598 & 0,607 & 0,644 & 0,640 \\
\hline Ins. chl. & & 2,38 & 0,79 & & 1,49 & 1,21 \\
\hline E.N.A. & 23,53 & 28,35 & 30,72 & 29,06 & 25,80 & 29,89 \\
\hline M.P.D. Bovin & 44,16 & 39,90 & 37,49 & 39,54 & 43,14 & 38,76 \\
\hline M.P.D. Porc & 46,06 & 41,65 & 39,16 & 41,30 & 45,05 & 40,48 \\
\hline M.P.D. Volaille & $40, \mathcal{T 3}$ & 36,78 & 34,57 & 36,47 & 39,78 & 35,74 \\
\hline T.D.N. Bovin & 81,30 & 81,06 & 77,22 & 75,55 & 77,34 & at,, 00 \\
\hline T.D.N. Pore & 84,32 & 77,95 & 80,86 & 78,44 & 81,38 & 81,88 \\
\hline U. F. Bovin & 1,10 & 1,09 & 1,02 & 1,00 & 1,02 & 1,09 \\
\hline U. F. Porc & 1,14 & 1.03 & 1,08 & 1,05 & $1, \infty 9$ & 1,10 \\
\hline E. M. Porc & 3457 & 3195 & 3315 & 3216 & 3337 & 3357 \\
\hline E. M. Volaille & 2966 & 2651 & 2790 & 2748 & 2790 & 2832 \\
\hline
\end{tabular}

1. Huilexie Blohorn (Abidjan 1961) - 2. Huilerie Blohorn (Abiajan 1962) - 3. Huilerie Blohorn (Abidjan 1964) 4. Hujierie Citec (Bobodioulasso 1960) - 5. Hujlerie Citec (Bobodioulasso 1964) - 6. Huilerie Citec (Bobodioulasso 1964).

matıères grasses, supérieures à 7 p. 100, sont un peu élevées pour des expellers. Toutefois, cet inconvénient, pour le stockage (rancissement des $M . G$.), est limité avec les usınes qui fonctionnent toute l'année; en effet, on peut s'assurer un ravitaillement en tourtecux, fraîchement préparés et donc de bonne qualıté, puisqu'on sait que les graisses rancissent et s'oxydent plus facilement dans le tourteau que dans la graine. Pour les usines qui fonctionnent temporarement, comme la C.I.T.E.C. de Bobodioulasso, les inconvénients sont certains : risques de rupture de stock, ce qui est grave pour le fabricant de concentrés, ou constitution de stocks, avec risque de baisse de qualité du produit et de pertes par les insectes prédateurs ou les rats.
Nous avons pu observer l'apparition du rancissement et le développement de l'acidité après stockage d'un tourteau fabriqué à Koulikoro (colonne 3 du tableau $X X \mid$ ) dont la teneur en $M$. G. est un peu élevée. Quelques semaines après sa fabrication, le taux d'acidité est de $0,30 \mathrm{~g}$ p. 100 d'acıde sulfurique. Après 5 mois de stockage à Dakar, où le produit était resté en souffrance, le taux d'acidité passait à 1,40 g p. 100 d'acide sulfurique, ce qui dépasse de très loin le taux toléré par les fabricants d'aliments du bétail.

\section{Tourfeau « exfraction» (Tableau XXI)}

Deux usines, en Afrique de l'Ouest francophone, extraient par solvant, l'huile des graines d'orachides. 
TURTESJ H० TEI

Tourteanx d'arachides "expellers" et "extraction"

\begin{tabular}{|c|c|c|c|c|c|}
\hline & 1 & 2 & 3 & 4 & 5 \\
\hline Humidité & 5,36 & 5,12 & 4,38 & 8,19 & 8,12 \\
\hline Matilère sèche & 94,64 & 94,88 & 95,02 & 91,81 & 91,88 \\
\hline M.P. B. & 51,60 & 53,40 & 48,82 & 49.78 & 52,41 \\
\hline Cellulose & 7,73 & 6,70 & 7,20 & 9,10 & 7,35 \\
\hline Mat. Brasses & 8,38 & 5,50 & 7,99 & 4,61 & 0,80 \\
\hline Mat. min. & 5,26 & 4,75 & 5,47 & 4,18 & 4,58 \\
\hline Calcium & 0,074 & 0,070 & 0,094 & 0,092 & 0,108 \\
\hline Fhosphore & 0,532 & 0,496 & 0,558 & 0,534 & 0,594 \\
\hline Ins, chl. & 1,38 & 0,85 & 1,29 & 0,22 & 0,35 \\
\hline E.N.A. & 21,67 & 24,53 & 25,54 & 24,15 & 26,74 \\
\hline M.P.D. Bovin & 46,44 & 48,06 & 43,94 & 44,80 & 47,17 \\
\hline M.P.D. Pore & 48,50 & 50,20 & 45,89 & 46,79 & 49,26 \\
\hline M.P.D. Volaille & 42,82 & 44,32 & 40,52 & 41.31 & 43,50 \\
\hline T.D.N. Bovin & 84,06 & 81,83 & 83,80 & 77,20 & 73,35 \\
\hline T.D.N. Porc & 87,96 & 85,89 & 84,96 & 82,15 & 78,38 \\
\hline J. F. Bovin & 1,13 & 1,08 & 1,12 & 1,01 & 0,94 \\
\hline J. F. Porc & 1,35 & 1,15 & \pm .15 & 1,10 & 1,04 \\
\hline E. H. Porc & 3606 & 3522 & 3483 & 3369 & 3214 \\
\hline E. M. Volacille & 3035 & 2970 & 3050 & 2775 & 2656 \\
\hline
\end{tabular}

1. Huilerie Maradi (Niger 1963) expeller - 2. Hullerie Maredt (Niger 1964) expeller - 3. Builerie Roultkoro (Mali 1964) expeller - 4. Huilerie Petersen (Dakar 1964) expeller - 5. Hullerie Lesieur (Dakar 1964) extraction

\section{Ce sont : LESIEUR - Afrique,}

\section{S. E.I. C.}

Une autre usine utilise, simultanément, les deux procédés: pressıon puis extraction (S. O.D.E.C.). Ces trois huleries sont implantées au Sénégal. Le tourteau obtenu par LESIEUR (le seul que nous ayons analysé), est de bonne qualité (colonne 5).

Autres sous-produits de l'arachide (Tableau XXII)

On írouve, sur de nombreux marchés des zones productrices d'arachides. des produits de fabrication artisanale ou familiale que l'on devrait qualıfier de pâte d'arachide deshulée plutôt que tourteau, car leur teneur en matières grasses reste très élevée, 23 à 24 p. 100, alors que la graine décortıquée en contient 47 à 48 p. 100.

Pour fabriquer ces tourleaux, on écrase au pilon les graınes décortiquées qui souvent, ont été grıllées au préalable pour faciliter le broyage. On obtient une pâte qui est mise à bouillır; I'huile surnage, elle est alors recueillie par écumage ef le résidu est mis à sécher.

Ces produits sont utılısés dans l'alımentation humarne comme condıments. Au Sénégal, ils sonf quelquefois donnés aux moutons de case qui aftergnent dans ce pays des prix astronomiques.

Les sons d'arachide (colonne 3) proviennent de la pellicule rouge qui recouvre la graine décortiquée, employée, dans ce cas, pour la 
TABTEAU $7^{\circ}$ XCII

Sutres sous-produits de l'arachide

\begin{tabular}{|c|c|c|c|}
\hline & 1 & 2 & 3 \\
\hline Humidzité & 4,78 & 6,75 & 7.38 \\
\hline Matière sèche & 95,22 & 93,25 & 92,62 \\
\hline M.P.B. & 42,78 & 44,45 & 16,76 \\
\hline Cellulose & 5,24 & 5,00 & 25,65 \\
\hline Mat. Exasses & 23,93 & 23,20 & 1,17 \\
\hline Mat. min. & 3,90 & 3,76 & 6,30 \\
\hline Calcium & 0,062 & 0,006 & 0,204 \\
\hline Phosphore & 0,402 & 0,452 & 0,168 \\
\hline Ins. che. & 0,86 & 0,41 & 2,85 \\
\hline E.N.A. & 19,37 & 16,84 & 42,74 \\
\hline M.P.D. Bovin & 38,44 & 40,00 & $11,23 ?$ \\
\hline M.P.D. Porc & 40,21 & 41,78 & $7,87 ?$ \\
\hline M.P.D. Volaille & 35,50 & 36,89 & \\
\hline T.D.N. Bovin & 104,0 & 101,0 & $53,99 ?$ \\
\hline T.D.N. Pore & 105,0 & 103,0 & $34,83 ?$ \\
\hline D. F. Bovin & 1,58 & 1,53 & $0,56 ?$ \\
\hline J. F. Porc & 1,48 & 1,45 & $0,27 ?$ \\
\hline D. M. Foro & 4305 & 4223 & $1428 ?$ \\
\hline E. M. Volaille & 3831 & 3774 & \\
\hline
\end{tabular}

1. Pâte ou tourteau artisanal (T1liabery, Niger) 2. Pâte ou tourteau artisanal (Maradi, Niger) -3 . Son deshuilé (Dakar)

fabrication des farines d'arachıde deshuilées destinées à l'alimentation humaine. Ce sont les ruminants qui exploitent au maximum ces produits. On peut, en particulier, les préconıser pour les vaches laitıères et les jeunes bovins, comme aliments de post-sevrage (richesse en matières protéiques digestibles). Le porc les exploite mal par suite, sans doute, de leur haute teneur en cellulose.

\section{Graines de coton et dérivés (Tableau XXIII)}

Les pays africains produisent des quantités de plus en plus importantes de coton-graine. Les installations d'égrenage se sont également développées ef les graines de coton sont disponibles, en quantités importantes, dans de nombreuses régions.

Ces graines sont, dans la plupart des cas, inutilısées ou brôlées pour fournir l'énergie à quelques usines, ou valorisées sous forme de devises, mals à des taux dérisoires, par l'exportation.

Elles constituent pourtant un aliment de grande valeur pour les bovins adultes, aınsi que le montre le tableau XXIII. Certains auteurs américains estiment que ces graines sont encore un aliment passable pour le porc lorsqu'elles contiennent moins de 24 p. 100 de cellulose (colonnes 1 et 2 ).

Dans les régions où elles sont disponıbles, elłes représentent l'alıment énergétique le moins cher pour les bovins. Par ailleurs, les ruminants sont beaucoup morns sensibles au gossypol que les porcs et surtout les volailles.

Une seule usine extrait l'huile de la graine de coton : celle de Niono (anciennement Office du Niger, au Mall). Elle obtient un tourteau de médiocre qualité par suite de la teneur trop élevée en matières grasses (colonne 5).

L'emploi de la graine de coton en alimentation animale n'a pas été assez vulgarisé dans les pays de l'Afrique de l'Ouest, bien que des essais aient été effectués avec succès, depuis longtemps, dans certaines stations administratives. II semble que les bovins adultes peuvent en accepter facilement $2 \mathrm{~kg}$ par jour et sans doute plus.

Tourteau de coprah et de palmiste (Tableau XXIV)

Coprah : colonnes 1, 2, 3, 4 du tableau XXIV.

L'usıne BLOHORN est la seule qui traite cette matière, en Afrique de l'Ouest francophone. Le tourteau obtenu semble, d'après sa composition, avoir une assez bonne valeur bromatologique. Il est cependant un peu trop riche en matières grasses. Les taux de M. P. B. et de cellulose sont corrects alors que le tourteau originaire de Nouvelle-Calédonie contient trop de ce polyoside.

En réalité, le coprah abtenu en Côte-d'Ivoire a une assez mauvaise réputation par suite des techniques défectueuses de séchage qui lui sont appliquées. Les graisses y rancissent vite et les moisissures s'y développent, paraît-il, très rapidement. 


\section{TABLAU N MII}

Graines de coton et dérivés

\begin{tabular}{|c|c|c|c|c|c|c|}
\hline & 1 & 2 & 3 & 4 & 5 & 6 \\
\hline Elumidité & 7,85 & 8,25 & 5,70 & 4,72 & 5,82 & 6,75 \\
\hline Matière sèche & 92,15 & 91.75 & 94,30 & 95,28 & 94,18 & 93,25 \\
\hline M.P.B. & 19,82 & 19,72 & 19,88 & 32,74 & 36,94 & $7, \infty$ \\
\hline Celluiose & 21,40 & 23.40 & 27,70 & 6.53 & 12,95 & 48,87 \\
\hline Nat. Erasses & 21,81 & 20,45 & 20,93 & 36,21 & 14,84 & 5,65 \\
\hline Mat. min. & 3,94 & 4,46 & 3,76 & 5,89 & 6,78 & 1,63 \\
\hline Calcium & 0,127 & 0,226 & 0,160 & 0,195 & 0,219 & 0,125 \\
\hline Phosphore & 0,575 & 0.653 & 0,483 & 0,824 & 0,933 & 0,142 \\
\hline Ins. chl. & 0,10 & 0,26 & 0,32 & 1,36 & 1,29 & 0,06 \\
\hline E.N.A. & 25,18 & 23,72 & 22,03 & 13.91 & 22,67 & 30,10 \\
\hline M.P.D. Bovin & 12,48 & 12,42 & 12,52 & & 29,18 & 0,40 \\
\hline M.P.D. Porc & 9,51 & 9,46 & $9.54 ?$ & & 30,29 & \\
\hline \multicolumn{7}{|l|}{ M.P.D. Voleille } \\
\hline T.D.N. Borln & 82,16 & 79,74 & 82,43 & & 93,65 & 38,60 \\
\hline T.D.N. Porc & 46,28 & 43,04 & $44,70 ?$ & & 85,35 & \\
\hline J. F. Bovin & 1,11 & 1,06 & 1.10 & & 1,32 & 0,26 \\
\hline U. P. Porc & 0,47 & 0,42 & $0,44 ?$ & & 1,15 & \\
\hline E. M. Porc & 1897 & 1764 & $1830 ?$ & & 3500 & \\
\hline E. M. Volaille & & & & & & \\
\hline
\end{tabular}

1. Graines A 333-57 - IRCP Bouaké (Cote d'Itoire) gossypol libre : 0,038 pour cent - 2. Grainea mono 63 - Iret Bougké (Cotte d'Ivoire) gossypol libre : traces - 3. Graines de' Niono (Meli) gossypol libre : 0,0074 pour cent. 4. Arandes de la graine 3 gossypol libre : 0,0074 pour cent - 5. Tourteau de Ia graine 3 gossypol libre : 0,0157 pour cent - 6. Coques de la sraine 3.

Polmiste : colonnes 5 et 6 du tableau XXIV.

Nous n'avons trouvé aucun renseignement concernant la digestibilité du tourteau de palmiste chez le porc.

Ce produit fabrıqué en toute petite quantifé à Abidjan est de qualité équivalente à ceux que l'on trouve alleurs, mais le taux de matières grasses est trop élevé.

\section{D. - PRODUITS DIVERS D'ORIGINE VÉGÉTALE}

\section{Produits végétaux divers (Tableau $X \times \mathrm{V}$ )}

Nous avons trouvé un certain nombre de produits végétaux; quelques-uns sonł déjà utilisés pour les animaux domestiques, en Afrique mass on connaît mal les qualités bromatologiques. Leur utilisation peut rendre des services dans certans cas.

\section{Colonne 1 - Jeunes feullies de Boobob.}

Ces feuılies, réduites à l'état de farıne, sont utilisées dans l'alimentation humaine pour la préparation des sauces, des condiments.

En Haute-Volta, elles apparaissent en pleıne saison sèche et nous pensons qu'elles pourraient, à ce moment, rendre de grands services dans l'alimentation des poussins, des veaux et des porcelets, par suite de leur bonne teneur pro- 
TABIEAD NO XXIV

Tourteaux de coprah et de palnoste

\begin{tabular}{|c|c|c|c|c|c|c|}
\hline & 1 & 2 & 3 & 4 & 5 & 6 \\
\hline Bunoiaité & 12,02 & 9,95 & 5,30 & 9,87 & 9,73 & 9,56 \\
\hline Matière sèche & 87,98 & 90,05 & 94,70 & 90,13 & 90,27 & 90,44 \\
\hline M.P.B. & 20,73 & 21,40 & 21,97 & 21,26 & 15,40 & 16,45 \\
\hline Cellulose & 10,38 & 8,15 & 12,30 & 23,85 & 19,22 & 21,35 \\
\hline Mat. Grasses & 7,65 & 13,25 & 10,60 & 9,90 & 13,73 & 9,23 \\
\hline Mat. min. & 9,06 & 6,55 & 8,02 & 5,37 & 3,52 & 4,00 \\
\hline Calcium & 0,140 & 0,078 & 0,067 & 0,232 & 0,343 & 0,273 \\
\hline Phosphore & 0,633 & 0,550 & 0,567 & 0,475 & 0,658 & 0,616 \\
\hline Ins. chl. & & & 2,93 & 1,80 & & 0,66 \\
\hline E.N.A. & 40,10 & 40,70 & 41,88 & 29,75 & 38,40 & 39,41 \\
\hline M.P.D. Bovin & 16,80 & 17,33 & 17,80 & 17,22 & 12,76 & 13,65 \\
\hline M.P.D. Porc & 15,13 & 15,63 & 16,04 & $15,52 ?$ & & \\
\hline M.P.D, volaille & 15,54 & 16,05 & 16,48 & & & \\
\hline T.D.N. Borin & $\tau_{1}, 44$ & 86,36 & 81,58 & 77,23 & 96,41 & 88,02 \\
\hline T.D.N. Porc & 71,27 & 81,79 & 80,49 & $74,72 ?$ & & \\
\hline U. F. Bovin & 0,91 & 1,14 & $i, 08$ & 1,02 & 1,39 & 1,23 \\
\hline J. F. Porc & 0,92 & 1,10 & 1,06 & $0,97 ?$ & 1 & \\
\hline E. M. Porc & 2920 & 3350 & 3300 & 3063 ? & & \\
\hline E. M. Volaille & 2465 & 2960 & 2836 & & & \\
\hline
\end{tabular}

1. Coprah - huilerie blohorn (Abıdjan 1961) - 2. Coprah - huilerie blohorn (Abidjan 1963) - 3. Coprah - Huilerie blohorn (Abidjan 1964) - 4. Coprsh (Nouvelle-Calédonie 1963) - 5 Palmisto - huilerie blohorn (Abidjan 1961) 6. Palmiste - huilerie blohorn (Abidjan 1964).

bable en vitamıne $A$, et du taux de calcium exceptionnellement élevé pour un végétal, observation déjà falte par les spécialistes de l'alimentation humaine de I'O. R. A.N.A.

\section{Colonne 2 - Farine de gousses de Néré.}

Les graines contenues dans les gousses de néré sont entourées d'une farine jaunâtre qui est quelquefois utilisée en alimentation humarne pour fabriquer des galettes, en mélange avec du miel, du lait caillé, etc...

Un élevage de porcs fort important de la Nıgeria en incorporait régulièrement dans la ration, suivant la formule appliquée également à la porcherie de MARADI: 1/3 tourteau d'arachide $1 / 3$ mélange sorgho + mil, 1/3 farine denéré.

D'après les chercheurs de f'O. R. A. N. A. la farine de néré serait riche en acide ascorbıque (vitamıne C).

\section{Colonne 4 - Tourfeau de sésame.}

II s'agit d'un produit fabriqué dans les mêmes conditions que nous avons décrites pour la pâte d'arachide.

Colonne 5 - Graines de Gonaklé.

Les bovins mangent cette graine d'acacia dont la gousse sert à fabriquer un produit utilısé 


\section{TABLEAO H' XTV}

Produits végétaux divers

\begin{tabular}{|c|c|c|c|c|c|c|}
\hline & 1 & 2 & 3 & 4 & 5 & 6 \\
\hline Fumiditté & 8,96 & 4,84 & 2,53 & 5,02 & 7,50 & 9,66 \\
\hline Matière aèche & 91,04 & 95,16 & 97,47 & 94,98 & 92,50 & 90,34 \\
\hline M.P.B. & 9,61 & 2,43 & 22,20 & 40,33 & 15,40 & 2,94 \\
\hline Cellulose & 14,35 & 12,47 & 4,60 & 7,19 & 21,15 & 21,80 \\
\hline Nat. grasses & 1,82 & 0,89 & 54,50 & 21,00 & 2,79 & 1,59 \\
\hline Mat. min. & 7,79 & 4,65 & 4,68 & 6,86 & 5,70 & 4,11 \\
\hline Calcium & 1,080 & 0,286 & 0,377 & & 0,588 & 0,123 \\
\hline Phosphore & 0,400 & 0,107 & 0,452 & & 0,215 & 0,132 \\
\hline Ins. chI. & 0,65 & 0,62 & 0,75 & & 0,59 & 0,48 \\
\hline E.N.A. & 57,47 & 74,72 & 11,49 & 19,60 & 47,46 & 59,90 \\
\hline M.P.D. Bovin & & & & 36,70 & & $0 ?$ \\
\hline M.P.D. Pore & 5,86 & $1,65 ?$ & & & & 0 ? \\
\hline M.P.D. Volainle & $7,11 ?$ & & & & & \\
\hline T.D.N. Borin & & & & 84,00 & & $65,39 ?$ \\
\hline T.D.N. Porc & 53,30 & $76,13 ?$ & & & & $63,20 ?$ \\
\hline U. F. Bovin & & & & 1,12 & & $0,78 ?$ \\
\hline U. F. Porc & 0,60 & $0,98 ?$ & & & & $0,78 ?$ \\
\hline E. M. Pore & 2180 & 3120 & & & & $2590 ?$ \\
\hline E. M. Volailue & $1530 ?$ & & & & & \\
\hline
\end{tabular}

1. Jeunea feutlles de Baobab (Haute-Volta) - 2. Farine de gousses de zèré de Nigeria (prélevée au Nigrer) 3. Graines de Sesame (Niger) - 4. Tourteau artisanal de Sesame (Haute-Volta) - 5. Graines de Gonakié (HauteVolta) - 6. Graines de Doum (Niger)

pour le tannage des currs. La coque de cette grane est extrêmement dure et nous n'avons pu vérifier si, non concassée, elle étalt attaquée par les sucs digestifs.

\section{Colonne 6 - Granes de Doum.}

Le doum est un palmier du genre Hyphaene, des régions sahélıennes. L'amande de la graine a une valeur énergétique assez intéressante pour ces régions particulièrement deshéritées, maıs elle est sı dure qu'elle ne peut être utılısée sans broyage, ce qui, dans ce milieu, est pratiquement impossıble à réaliser à bas prix.

\section{Sous-produts divers} de l'industrie ogricole (Tableau XXVI)

Colonnes 1 et 2 - Cacao.

La cabosse est le fruit du cacaoyer ; elle pèse en moyenne, à l'état frais, de 400 à $500 \mathrm{~g}$. Elle est essentiellement constituée par un parenchyme mucl lagineux au milieu duquel on trouve, serrées, les granes ou fèves de cacao, noyées dans une pulpe dont nous donnons la composition en colonne 1. Maıs cette pulpe disparaît en grande partie au cours de la fermentation que subissent les graines avant d'être livrées au commerce ef 
TABTEAU NO XNVI

Sous-produits divers de l'industrle egrioole

\begin{tabular}{|c|c|c|c|c|}
\hline & 1 & 2 & 3 & 4 \\
\hline Humidité & 12,65 & 8,81 & 8,58 & 10,04 \\
\hline Matière sèche & 87,35 & 91,19 & 91,42 & 89,96 \\
\hline M.P.B. & 18,71 & 7,92 & 3,62 & 1,38 \\
\hline Cellulose & $1 \uparrow, 44$ & 21,60 & 16,85 & 35,85 \\
\hline Mat. grasses & 14,84 & 0,67 & 0,90 & 0,50 \\
\hline Mat. min. & 14,67 & 8,50 & 4,10 & 2,42 \\
\hline Calcium & 0,524 & 0,204 & 0,224 & 0,068 \\
\hline Phosphore & 0,593 & 0,147 & 0,195 & 0,039 \\
\hline Ins, chl. & 0,12 & 0,05 & 0,49 & 1,35 \\
\hline B.N.A. & 27,69 & 52,50 & 65,96 & 49,61 \\
\hline M.P.D. Bovin & 4,50 & 0,87 & 0 & 0 \\
\hline H.P.D. Pore & $4,00 ?$ & & $1,50 ?$ & \\
\hline \multicolumn{5}{|l|}{ M.P.D. Volaille } \\
\hline T.D.N. Bovin & 52,84 & 51,71 & 67,04 & 42,36 \\
\hline T.D.N. Porc & $42,00 ?$ & & $74,17 ?$ & \\
\hline U. F. Bovin & 0.56 & 0,52 & 0,81 & 0,34 \\
\hline D. F. Pore & $0,45 ?$ & & $0,96 ?$ & \\
\hline E. H. Porc & $1720 ?$ & & $3040 ?$ & \\
\hline E. M. Volatill & & & & \\
\hline
\end{tabular}

1. Résidug de la pulpe de cabosse de crcao (Cotte d'Iroire) - 2. Cabosge de cacao (Cote d'Itoire) - 3. Déchets de la fabrication de congerves d'ananas (Cotte d'Itroire) - 4. Bagasge de canne à sucre (Kall).

\begin{tabular}{|c|c|c|c|c|}
\hline & Glucides hydrolysables & Sucres réducteurg & Sucres totaux & Acidité en gr. de S04 He \\
\hline 3 & 8,10 & 19,20 & 27,30 & 1,88 \\
\hline 4 & 21,30 & 10,25 & 31,55 & 0,28 \\
\hline
\end{tabular}

il reste surtout ce parenchyme mucilagineux qui, séché, a reçu le nom de coque de cacao.

D'après MORRISSON, la quantité de matières protéıques digestıbles est négligeable, mais au point de vue énergétique et pour less bovins, les coques de cacao équivalent à la moitié de la valeur du mais.

Avant toute utilisation, Il faudrait vérıfier les teneurs en caféine et théobromıne.

Colonne 3 - Ananas.

L'utilisation des déchets de conserverie, pour l'alimentation des bovins ou des porcs, doit être précédée d'une neutralısation de l'acidité, sınon on risque de graves troubles intestinaux déjà observés à Abidjan. Même neutralisé, le produit devra être utilisé frais. La neutralisation pourra être effectuée par exemple par de la chaux. Pour $1,88 \mathrm{~g}$ d'acidité, il est facile de calculer qu'il faut environ $15 \mathrm{~g}$ de chaux par $\mathrm{kg}$ de déchet.

Pour le porc, on ne dépassera pas $1 / 3$ de la ration en produit séché tel qu'।l est rapporté dans le tableau. Pour les bovins on peut aller jusqu'à 60 p. 100. 
TAFTRAO NO RXII

Farines de poissons de mer

\begin{tabular}{|c|c|c|c|c|c|}
\hline & 1 & 2 & 3 & 4 & 5 \\
\hline Humidité & 9,08 & 6,17 & 8,72 & 7,27 & 7,80 \\
\hline Watière sèche & 90,92 & 93,83 & 91,28 & 92,73 & 92,20 \\
\hline M.P.B. & 60,99 & 62,45 & 60,00 & 25,37 & $65, \infty 0$ \\
\hline \multicolumn{6}{|l|}{ Cellulose } \\
\hline Mat. ETasses & 3,65 & 8,47 & 7,14 & 7,03 & 2,25 \\
\hline Mat. min. & 20,11 & 20,19 & 20,23 & 47,00 & 14,40 \\
\hline Calcium & 6,000 & 5,632 & 6,080 & 11,92 & 3,520 \\
\hline Phosphore & 3,320 & 3,080 & 3,290 & 0,766 & 2,470 \\
\hline Ins. Chl. & 0,79 & 2,28 & 0,84 & 14,10 & 0,60 \\
\hline \multicolumn{6}{|l|}{ E.N.A. } \\
\hline M.P.D. Bovin & 46,35 & 47,46 & 45,60 & & 49,40 \\
\hline M.P.D. Forc & 57,94 & 59,33 & 57,00 & & 60,45 \\
\hline M.P.D. Volaille & 55,50 & 56,82 & 54,60 & & 59,15 \\
\hline T.D.N. Bovin & 54,32 & 65,96 & 61,18 & & 54,30 \\
\hline T.D.N. Porc & 66,33 & 78,75 & 73,42 & & 64,54 \\
\hline U. F. Bovin & 0,57 & 0,78 & 0,70 & & 0,56 \\
\hline U. F. Pore & 0,82 & 1,03 & 0,95 & & 0,79 \\
\hline E. M. Porc & 2719 & 3220 & 3010 & & 2646 \\
\hline E. M. Volatlle & 2457 & 2928 & 2723 & & 2532 \\
\hline
\end{tabular}

1. Farine de déchets de thon - Dakar (mai 64) - 2. Farine de poissons entiers (Clupeides) - Dakar (mal 64) -

3. Farfne de déchets de thon - Dakar (janvier 64) - 4. Farine artisanale - Rivage Aladjan - Cote d'Ivoire -

5. Farlne du Pérou - prélevéè̀ Cotonou.

Colonne 4 -Canne d sucre.

Nous n'avons pas eu l'occasıon de prélever de «bouts blancs » de canne, mais seulement des bagasses qui sont constıtuées par ce qui reste de la tige lorsqu'elle a été passée au concasseur qui en extralt lo sève sucrée. D'après SCHNEIDER, le coefficient de digestibilité de la cellulose est de: 0,58 chez les bovins.

\section{E. - PRODUITS D'ORIGINE ANIMALE}

Farines de poissons de mer (Tableau XXVII)

Il y a eu, ces dernières années, quelques fabrıcations artisanales de farınes de poisson, à Dakar en particulier. Elles ont toutes cessé leur activité, pour deux ratsons essentielles : irrégula- rité de l'approvisionnement en matières premières, mauvaise qualité des produits finis.

Depuis le début de l'année 1964, fonctionne d̀ Dakar une petite usine qui fabrique principalement de la farine de déchets de thon et éventuellement de la farine de poissons entiers (colonnes 1. 2 et 3).

Si on tient compte de la matière première, qui est un déchet de conserverie (viscères, têtes, etc...). le produit obtenu peut être qualıfié de bon.

La farıne 1, fabriquée en ma 1964 et analysée en juin, titrait en acidité $0,70 \mathrm{~g}$ d'acide sulfurique. La farine 3, fabriquée en janvier 1964 et analysée en juin, titrait $0,74 \mathrm{~g}$. La farıne convenablement séchée est mise dans des sacs de papier fort, 
TARIFAUT NNO KRVIII

Farines de poissons d'eau douce

\begin{tabular}{|c|c|c|c|c|}
\hline & 1 & 2 & 3 & 4 \\
\hline Humidité & 4,98 & 6,12 & 6,80 & 6,11 \\
\hline Matière sèche & 95,02 & 93,88 & 93,20 & 93,89 \\
\hline $\mathrm{M}_{*} \mathrm{P}_{\bullet} \mathrm{B}$ & 63,25 & 59,15 & 61,25 & 56,35 \\
\hline \multicolumn{5}{|l|}{ Cellulose } \\
\hline Mat. grasses & 10,14 & 8,19 & 8,48 & 11,51 \\
\hline Mat. min. & 19,30 & 25,33 & 23,45 & 20,37 \\
\hline Calcium & $4, \infty$ & 7,200 & 7,824 & 5,600 \\
\hline Phosphore & 2,41 & 3,790 & 3,893 & 3,110 \\
\hline Ins. ChI. & 4,71 & 1,95 & 1,17 & 1,43 \\
\hline \multicolumn{5}{|l|}{ E.N*A. } \\
\hline M.P.D. Bovin & 48,07 & 44,95 & 46,55 & 42,81 \\
\hline M.P.D. Porc & 59,45 & 53,83 & 55,73 & 52,95 \\
\hline M.P.D. Volaille & 57,56 & 53,83 & 55,73 & $5 t, 26$ \\
\hline T.D.N. Bovin & 80,17 & 62,80 & 65,03 & 66,90 \\
\hline T.D.N. Pore & 83,40 & 67,82 & 75,24 & 79,98 \\
\hline U. F. Bovin & 1,05 & 0,71 & 0,77 & 0,94 \\
\hline U. F. Pore & 1,11 & 0,84 & 0,97 & 1,05 \\
\hline E. M. Porc & 3419 & 2780 & 3084 & 3279 \\
\hline E. M. Volaille & 3114 & 2543 & 2896 & 2978 \\
\hline
\end{tabular}

1. Farine artiganale - poisson du fleuve Niger - Tillabery (Niger) - 2. Farine prélevée à Ouggadougou - Fabriquée à partir de saisies. Origine du poisson inconnue - 3. Idem à 2. - 4. Farine artisanale - poisson du fleuve Niger - Sotube (Mali) acidité : 2,96 pour cent de $50^{4} \mathrm{H}^{2}$.

eux-mêmes enfermés dans des sacs de jute goudronné. Il semble que ce conditionnement donne satısfaction pour le stockage.

Les teneurs en calcium et phosphore sont bonnes.

Nous avons prélevé à Cotonou une farine de poissons entiers, originaire du Pérou ef incorporée dans des concentrés volailles. C'est une farine de bonne qualité, mass elle ne peut rendre les services de la farine de déchets de thon par suite de son prix : elle revenait, en avril 1964 , à 88-90 F magasin Cotonou, alors que la farine de déchets de thon pourrait être vendue 30-35 F F. O. B. Dakar.

En colonne 4, on trouvera une farine de fabrication artisanale. Elle est de mauvaise qualité : la haute teneur en calcium montre qu'il s'agit d'une farine fabrıquée avec des déchets composés surtout d'arêtes, de têtes et de nageoires. Enfin, le pourcentage d'insoluble chlorhydrique lasse supposer que les opérations de dépeçage et de séchage ont été effectuées sur le sable des plages. La teneur en M. P. B. est faible et le coefficient de digestibilité de ces protéines doit être également assez bas par suite de la proportion inhabituelle de matières minérales totales, ce coefficient baissant sensiblement lorsque leur taux s'élève.

\section{Farines de poissons d'eau douce (Tableau XXVIII)}

Il ne s'agit pas ici de sous-produits fabriqués avec des déchets, mais de farınes fabriquées. 
TABLEAU Ho $\mathrm{NDX}$

Farines de viarde et d'os

\begin{tabular}{|c|c|c|c|c|c|c|}
\hline & 1 & 2 & 3 & 4 & 5 & 6 \\
\hline Humiditis & 6,54 & 5,76 & 5,80 & 19,98 & 6,61 & 8,10 \\
\hline Matière sèche & 93,46 & 94,24 & 94,20 & 80,02 & 93,39 & 91,90 \\
\hline M.P.B. & 54,68 & 56,12 & 59,19 & 53,06 & 31,92 & 30,62 \\
\hline \multicolumn{7}{|l|}{ Cellulose } \\
\hline Mat. Erasses & 11,80 & 17,98 & 15,23 & 10,70 & 7,34 & 5,93 \\
\hline Mat. min. & 26,92 & 15,89 & 15,24 & 10,67 & 52,60 & 52,85 \\
\hline Calcium & 8,87 & 4,264 & 4,160 & 2,74 & 18,08 & 21,12 \\
\hline Phosphore & 4,48 & 2,630 & 2,304 & & 8,64 & 8,68 \\
\hline Ins. Chl, & 0,38 & 1,22 & 1,13 & & 0,38 & 0,63 \\
\hline E.N.A. & 0,16 & 4,25 & 4,54 & 5,59 & 1,53 & 1,60 \\
\hline \multicolumn{7}{|l|}{ M.P.D. Bovin } \\
\hline M.P.D. Porc & 48,66 & 49,94 & 52,68 & 46,16 & 29,37 & 28,17 \\
\hline M.P.D. Volaille & 21,87 & 22,45 & 23,67 & 48,28 & 12,77 & 12,25 \\
\hline \multicolumn{7}{|l|}{ T.D.N. Bovin } \\
\hline T.D.N. Pore & 75,21 & 90,40 & 86,90 & 70,23 & 47,34 & 44,30 \\
\hline \multicolumn{7}{|l|}{ U. F, Bovin } \\
\hline U. F. POrC & 0,94 & 1,23 & 1,17 & 0,93 & 0,48 & 0,44 \\
\hline E. M. Pore & 3085 & 3700 & 3562 & 2880 & 1940 & 1816 \\
\hline E. M. Volaille & 1880 & 2450 & 2250 & 2772 & $\$ 138$ & 1000 \\
\hline
\end{tabular}

1. Farine de viande - Ouagadougou (1963) - 2. Farine de viande - Guagadougou (1963) - 3. Farine de viande Oungadougou (1964) - 4, Farine de viende - Abiajan (1964) - 5. Farine d'os - Oragadougou (1963) - 6. Farine d'os - Ouagadougou (1964).

soit avec des poissons entiers séchés survant les techniques artisanales (colonnes 1 et 4), soit avec du poisson frals salsi et transformé en farine d̀ l'usine de l'abattorr de Ouagadougou (colonnes 2 et 3).

La présence de très nombreux insectes dans la farine 4 et son taux d'acidité très élevé, montrent que les conditions de stockage étalent mauvaises.

Habituellement, les poissons séchés sont simplement emballés dans des nattes dans lesquelles ils restent jusqu'à la vente au détasl.

\section{Forines de viande ef d'os (Tableau XXIX)}

Nous avons trouvé à Ouagadougou la seule usıne véritable fabriquant de la farine de viande ef d'os avec des matières premières provenant de l'abatioir.

En colonne 1, Il s'agit d'une farine osseuse.

En colonnes 2 et 3 , farines de viande sans os. En colonnes 5 et 6 , farines d'os vert.

Les prodults sont de très bonne qualıté si on en juge par la composition, avec une teneur un peu élevée en matıères grasses pour les trois premiers.

\section{Farine de sang (Tableau $X X X$ )}

Les colonnes 1 et 2 se rapportent à des prodults fabriqués à l'usine d'Ouagadougou dans les mêmes conditions que la farıne de viande. Ce sont les caillots de sang qui sont mis dans les 


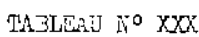

Farnes de sanz

\begin{tabular}{|c|c|c|c|}
\hline & 1 & 2 & 3 \\
\hline Humiditó & 7,55 & 8,05 & 9,85 \\
\hline latière sł̀che & 92,45 & 91,95 & 90,15 \\
\hline M.P.B. & 85,90 & 82,68 & 42,75 \\
\hline Cellulose & & & 2,95 \\
\hline Het. crasses & 0,56 & 0,62 & 6,93 \\
\hline Mat. min. & 5,19 & 5,35 & 13,65 \\
\hline Calcium & 0,200 & 0,248 & 3,52 \\
\hline Phosphore & 0,133 & 0,170 & 1,85 \\
\hline Ins. chl. & 1,21 & 1,94 & 1,43 \\
\hline E.NaA. & 0,15 & 2,80 & 23,97 \\
\hline \multicolumn{4}{|l|}{ I.P.D. Bovin } \\
\hline Il.P.D. Porc & 67,00 & 64,5 & \\
\hline H.P.D. Voleille & 68,72 & 66,14 & \\
\hline \multicolumn{4}{|l|}{ T.D.N. Bovin } \\
\hline T.D.N. Porc & 67,00 & 64,5 & \\
\hline \multicolumn{4}{|l|}{ J. F, Bovin } \\
\hline U. F. Porc & 0,83 & 0,79 & \\
\hline E. II. Porc & 2747 & 2644 & \\
\hline E. M. Volaille & 2687 & 2594 & \\
\hline
\end{tabular}

1. Farine de caillot - Ouagadougou (1963)

2. Farine de caillot - Oragadougou (1964)

3. Mélange : issues de mais et sanf entier - Bouaké (Cote d'Iroire). cuiseurs. Il y a donc perte presque intégrale du sérum ; il est impossible dans ces conditions de comparer l'efficacité protélque de cette farine de caillots avec les farines de sang habituelles. Maıs la technıque appliquée r'est pas critiquable car elle permet d'utiliser le même appareıl pour les viandes et le sang : une unité classique de fabrication de farine de sang ne serait pas rentable.

Le produit, dont la composition est consıgnée en colonne 3, a été fabriqué par un éleveur de porcs ; c'est un mélange comprenant 10 parties de son de maîs provenant d'un moulin artisanal, et 90 parties de sang entier bovilli. Le tout est homogénéssé et mis à sécher. Au point de vue bromatologique, il est certain que ce produif n'est bon que s'il est employé peu après sa fabrication car la conservation prolongée doit être difficile; le sang complémente très bien les protides du son de mas qui sont carencés en lysıne.

\section{Produits divers d'origıne anımale (Tableau $X X X \mid$ )}

Ces fabrications de petite importance peuvent rendre localement de grands services si les techniques appliquées sont convenables.

C'est ainsi que la farme de crabe de la colonne 2 a une composition voisine des farines homologues industrielles de l'Ouest des Etats-Unıs, sauf la teneur en matières minérales qui est trop élevée. Le pourcentage de calcium et de phosphore y est normal, mais la silıce se trouve d̀ un niveau trop élevé par suite du séchage de la matière première qui se fait sur sable.

\section{SUMMARY}

\section{Bromatological values of 150 west african foods}

A number of products originating in francophone West Africa, and adaptable to animal feeding, were analysed and studied from the technic, economıc, social point of view of their utilization.

These products belong to the following categories :

grains and by-products,

other essentially glucidic plants,

oll seeds and their by-products,

various vegetable products.

This study is to allow a more exact calculation of the rations. 


\section{TABLEAU NO XXXI}

Produits aiverg d'origine aninale

\begin{tabular}{|c|c|c|c|c|c|}
\hline & 1 & 2 & 3 & 4 & 5 \\
\hline Humidité & 7,17 & 12,05 & $7, \infty$ & 2,78 & 0.54 \\
\hline Matière sèche & 92,83 & 87,95 & 93,00 & 97,22 & 99,46 \\
\hline M.P.B. & 32,78 & 31,16 & 62,16 & & \\
\hline \multicolumn{6}{|l|}{ Cellutose } \\
\hline Mat. gresses & 0,15 & 7,75 & 10,36 & & \\
\hline Mat. min. & 8,14 & 39,48 & 4,60 & 94,50 & 96,63 \\
\hline Celcivin & 1,266 & 12,80 & 0,177 & 33,46 & 35,58 \\
\hline Phospore & 0,952 & 0,803 & 0,418 & 16,14 & 0,047 \\
\hline Ins, chl. , & 0,07 & & 1,09 & 1,27 & 4,98 \\
\hline E.N.A. & 51,76 & 9,56 & 16,08 & & \\
\hline \multicolumn{6}{|l|}{ M.P.D. Bovin } \\
\hline M.P.D. Porc & 32,12 & & & & \\
\hline M.P.D. Volaille & 29,50 & & & & \\
\hline \multicolumn{6}{|l|}{ T.D.N. Bovin } \\
\hline \multicolumn{6}{|l|}{ T.D.N. Porc } \\
\hline \multicolumn{6}{|l|}{ U. F. Bovin } \\
\hline \multicolumn{6}{|l|}{ U. F. Porc } \\
\hline E. M. Porc & 3500 & & & & \\
\hline E. M. Volaille & 2780 & 1900 & & & \\
\hline
\end{tabular}

1. Iait en poudre - origine U.S.A. - prélevé à Cotonou. Acidité : 0,195 gr. de SO4H² - 2. Farine de crabe artisanale - Rivage Alladian (Cotte d'Ivoire) - 3. Farine de sauterelles grillées - Maradi (Niger) - 4. Poudre d'os calcinés - Maradi (Niger) - 5. Poudre de coquille d'huitres - Cotonou.

\section{RESUMEN}

\section{Valores bromatologicas de 150 alimentos del oeste africano}

Se analizaron y se estudiaron, consıderando los puntos de visła técnico, economico y social de su utılızación, un cierto número de productos orıginarıos de los paises francófonos de Africa del Oeste, y podiendo utrlizarse en alimentación animal.

Estos productos pertenecen a las categorıas sıguientes

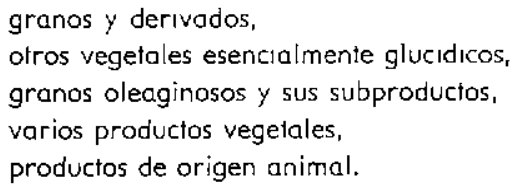

Este estudio debe permitir un calculo más justo de las raciones alımentıcias. 


\section{BIBLIOGRAPHIE}

ADRIAN (J.) et JACQUOT (R.). - Les sorghos et les mils en alimentation humaine et animale. Paris, Vigot, 1964.

CRAPLET (G.). - Aliments et alimentation des animaux domestiques. Paris, Vigot, 1955.

DAUMAS (R.). - Le bananier dans l'alimentation du bétail. Bull. Madagascar, 1962, 194: 623-32.

FRAPS (G. S.). - Practical Applications of Productive Energy Values to problems concerning Feeds and Feeding. Proc. Am. So, Anim. Prod., 1937, $30: 20-26$.

FRAPS (G. S.). - Composition and productive energy of poultry, feeds and rations. Bull. Tex. agrc. Exp. Stn, 1946 (678).

Inst. Prof. Contrôle et Rech. Scient. des Ind. Alim. animale - Méthodes d'analyse des alıments pour les animaux.

Journal Officiel R.F. - du 24-11-60. — Arrêté du 7-11-60.

LEROY $(A, M$.). - Utilisation de l'énergie des aliments. Ann. Zootech., 1954, 4, 337.
LEROY (A. M.). - L'Elevage rationnel des animaux domestiques. Paris, 1958.

LEROY (A. M.). - Utilisation de l'énergie des aliments par les volailles. Proc. 2nd Symp. on Energy Metabolısm. Wageningen. Sept.1961, p. 285.

LEROY (A. M.), FRANÇOIS (A.), MAITREJEAN (N.) ef PEROUNE (B.). - Contribution à l'étude de la composition chimique et de la valeur fourragère des aliments des animaux.

MORRISON (F. B.). - Feeds and Feeding. Morrison Publ. Cy, N-Y. 1946, 20 éd.

$$
\gg \quad \text { idem. - 1959, } 22 \text { éd. }
$$

SCHARRER (K.) et KURSCHNER (K.). - Bledermanns Z. 1931, 3, 302.

SCHNEIDER (B. N.), - Feeds of the World. Agr. Exp. St. Morgantown, 1947.

TITUS (H. W.). - Practical Feeding of Poultry in « Food and life 》. - 1939, 819.

TITUS (H. W.). - The Scientific feeding of chickens. Danville, Illinois, The Interstate, 1955. 